\title{
Prevalence of anemia and deficiency of iron, folic acid, and zinc in children younger than 2 years of age who use the health services provided by the Mexican Social Security Institute Ximena Duque1, Sergio Flores-Hernández ${ }^{1}$, Samuel Flores-Huerta ${ }^{2}$, Ignacio Méndez-Ramírez ${ }^{3}$, Sergio Muñoz ${ }^{4}$, Bernardo Turnbull1 ${ }^{1}$, Gloria Martínez-Andrade ${ }^{1}$, Rosa I Ramos ${ }^{1}$, Marco González-Unzaga ${ }^{1}$, María E Mendoza ${ }^{1}$ and Homero Martínez ${ }^{* 5,6}$
}

\begin{abstract}
Address: ${ }^{1}$ Unidad de Investigación en Epidemiología Nutricional, Instituto Mexicano del Seguro Social, Mexico D.F., Mexico, ${ }^{2}$ Departamento de Salud Comunitaria, Hospital Infantil de México "Federico Gómez", Mexico D.F., Mexico, ${ }^{3}$ Instituto de Investigaciones en Matemáticas Aplicadas y en Sistemas, Universidad Nacional Autónoma de México, Mexico D.F., Mexico, ${ }^{4}$ Facultad de Medicina, Universidad de la Frontera, Temuco, Chile, ${ }^{5}$ RAND, Santa Monica, CA, USA and ${ }^{6}$ Dirección de Investigación Médica, Hospital Infantil de México "Federico Gómez", Mexico D.F., Mexico

Email: Ximena Duque - ximena.duque@imss.gob.mx; Sergio Flores-Hernández - sergio.flores@imss.gob.mx; Samuel Flores-

Huerta - floreshuertamd@yahoo.com; Ignacio Méndez-Ramírez - imendez@servidor.unam.mx; Sergio Muñoz - munozs@ufro.cl; Bernardo Turnbull - bernardo.turnbull@imss.gob.mx; Gloria Martínez-Andrade - gloria.martineza@imss.gob.mx;

Rosa I Ramos - rosa.ramosh@imss.gob.mx; Marco González-Unzaga - marcounzaga@gmail.com;

María E Mendoza - mendoza_ma13@hotmail.com; Homero Martínez* - homero@rand.org

* Corresponding author
\end{abstract}

Published: 30 November 2007

BMC Public Health 2007, 7:345 doi:10.1 186/147I-2458-7-345

This article is available from: http://www.biomedcentral.com//47/-2458/7/345

(C) 2007 Duque et al; licensee BioMed Central Ltd.

This is an Open Access article distributed under the terms of the Creative Commons Attribution License (http://creativecommons.org/licenses/by/2.0), which permits unrestricted use, distribution, and reproduction in any medium, provided the original work is properly cited.
Received: 2I March 2007

Accepted: 30 November 2007

\begin{abstract}
Background: In Mexico, as in other developing countries, micronutrient deficiencies are common in infants between 6 and 24 months of age and are an important public health problem. The objective of this study was to determine the prevalence of anemia and of iron, folic acid, and zinc deficiencies in Mexican children under 2 years of age who use the health care services provided by the Mexican Institute for Social Security (IMSS).
\end{abstract}

Methods: A nationwide survey was conducted with a representative sample of children younger than 2 years of age, beneficiaries, and users of health care services provided by IMSS through its regular regimen (located in urban populations) and its Oportunidades program (services offered in rural areas). A subsample of 4,955 clinically healthy children was studied to determine their micronutrient status. A venous blood sample was drawn to determine hemoglobin, serum ferritin, percent of transferrin saturation, zinc, and folic acid. Descriptive statistics include point estimates and $95 \%$ confidence intervals for the sample and projections for the larger population from which the sample was drawn.

Results: Twenty percent of children younger than 2 years of age had anemia, and $27.8 \%$ (rural) to $32.6 \%$ (urban) had iron deficiency; more than $50 \%$ of anemia was not associated with low ferritin concentrations. Iron stores were more depleted as age increased. Low serum zinc and folic acid deficiencies were $28 \%$ and $10 \%$, respectively, in the urban areas, and $13 \%$ and $8 \%$, respectively, in rural areas. The prevalence of simultaneous iron and zinc deficiencies was $9.2 \%$ and $2.7 \%$ in urban 
and rural areas. Children with anemia have higher percentages of folic acid deficiency than children with normal iron status.

Conclusion: Iron and zinc deficiencies constitute the principal micronutrient deficiencies in Mexican children younger than 2 years old who use the health care services provided by IMSS. Anemia not associated with low ferritin values was more prevalent than iron-deficiency anemia. The presence of micronutrient deficiencies at this early age calls for effective preventive public nutrition programs to address them.

\section{Background}

The first 2 years of life are crucial for children's present and future health and nutritional status and, more specifically, for their mental, physical, and emotional development. In the last decade, micronutrient deficiencies have received much attention as it has been demonstrated that even subclinical states, such as mild iron deficiency or low concentrations of zinc, are associated with functional outcomes [1]. These include, among others, impaired psychomotor development $[2,3]$, decreased work capacity, diminished immunological response, and linear growth retardation [4-6]. Likewise, mild zinc deficiency has a negative influence on growth and development and increases the risk of diarrhea and acute respiratory infections [5,79]. Folic acid deficiency affects erythropoiesis, which, in turn, may be responsible for macrocytic anemia [10].

In Mexico, as in other developing countries, micronutrient deficiencies occur mainly between 6 and 24 months of age and are a significant public health problem [11-15]. Children under 2 have increased nutritional requirements because of their growth spurt, which often leads to a negative nutrient balance $[16,17]$. Preterm babies, as well as those born small for gestational age, are particularly vulnerable to iron deficiency in their first months [17]. During the first year of life, another risk factor for nutritional deficiencies occurs from inadequate complementary feeding practices. These are characterized by consumption of foods with low amounts of bioavailable micronutrients as well as with inhibitors of their absorption, and the practices often extend up to the age of 2 years. In the cases of iron and zinc, risk of inadequate intake is increased in countries where diets at this age include cereals with a high content of phytic acid coupled with low intake of animal foods $[1,7]$.

According to results from the National Nutrition Survey (NNS) in Mexico, carried out in 1999 (NNS-99), the highest prevalence of anemia was found in children between the ages of 12 and 24 months (48.9\%); there was a slightly higher prevalence in rural areas (52.9\%) as compared to urban ones $(46.8 \%)$ [15]. In children under 5 years old, $35 \%$ of anemia cases were not associated with iron deficiency but rather with one or more vitamin deficiencies [13]. In children between 1 and 2, 66.6\% of children had iron deficiency, as estimated by the percentage of transferrin saturation [13]. Folic acid deficiency was estimated in $8.8 \%$ of children under 2 and zinc deficiency in 34\% $[13,14]$. However, NNS-99 was not designed to be representative of children under 2 years old, so results for this age group are based on very small sample sizes.

When the study was conducted, the population of Mexico was approximately 100 million people. More than half of them were right-holders of the Instituto Mexicano del Seguro Social-IMSS (Mexican Institute for Social Security). Through two branches, IMSS provides social, welfare, and medical benefits for nongovernment employees and their direct dependents as well as for the underserved population living in rural or semi-rural areas. The regular regimen (RR) provides care to salaried employees, whereas IMSS-Oportunidades (IO) provides care to those in underserved, rural/semi-rural areas.

The present survey was carried out in primary health carelevel facilities, which offer services for infants and children such as medical care for common childhood illnesses (i.e., the common cold, acute diarrhea) as well as well-baby clinic, including growth monitoring, promotion and support of breast-feeding, and primary preventive actions (i.e., vaccination). Additionally, several government-sponsored programs geared to the underserved population are offered through IO program like distribution of fortified foods for pregnant women, infants, and malnourished children. In communities where IO operated, the whole community is a beneficiary of this program and no other public medical services are available, making it unlikely that people living in those communities will use other services. Also, IO has a strong component of home visits and community development activities, which tend to strengthen the relationship between health providers and community members.

This study was commissioned by the IMSS to learn about the prevalence of anemia and micronutrient deficiencies, particularly of iron, folic acid, and zinc, in children under 2 years of age; it was part of a larger study to document the general health and nutritional status of this age group. To this end, we carried out a national survey, drawing two representative samples of children under 2 years of age, 
beneficiaries of RR and IO. For each of these populations, the sampling frame was designed to be representative of the four regions in which the country has been divided by other national surveys, which take into consideration differences in socioeconomic development (i.e., North, Center, South, and Mexico City (the nation's capital) with its surrounding periurban area, which includes the Federal District and some municipalities of the surrounding State of Mexico); IO does not operate in this last region, as no major semi-rural or indigenous population lives there. Within each of these regions, RR is based in urban areas, while IO provides its services in the rural/semi-rural areas. We expect that the results of this survey will provide the basis for public health interventions and program strengthening, oriented to alleviate and prevent micronutrient deficiencies in the target population.

\section{Methods}

The survey was carried out between 1999 and 2001 [18]. From this larger study, which included 35,997 children younger than 24 months of age, a subsample was selected to determine micronutrient status to assess the prevalence of iron, folic acid, zinc deficiency, and anemia. Regarding ethical considerations, the Institutional Research Review Board approved the protocol study and, the informed consent letter was signed by mothers of the children in the subsample. The larger sample followed a stratified, twostaged random model by which we obtained a representative sample for each of the two care regimens as well as for each of the four regions of the country that have been used by other national surveys in the past. The sampling frame from which primary sampling units were selected was drawn from a list of all primary health care units in the country, which included 1,160 family medicine units (FMU) for RR and 3,367 rural medical units (RMU) for IO. FMUs were organized by region and number of attending physicians in each of them; RMUs were organized by region and number of children under 2 living in the communities where these units were located. Secondary sampling units were drawn from the children younger than 2 years old who attended the out-patient clinic, vaccination clinic, and well-baby clinic, all based in the primary health care units already described.

\section{Stratification of primary sampling units}

In RR, we defined five strata based on the number of physicians in each FMU. In each region, the sample size for each stratum was proportionally distributed by the number of children under 2 years of age, according to the 1997 census. In IO, we defined three strata, according to the number of children under 2 who lived in communities where the RMUs were located. In each region, sample size in each stratum was proportionally distributed by the number of children younger than 2 registered in 1998. In each stratum, we randomly selected one or more primary sampling units to complete the required sample size.

The sample size for the whole study was calculated based on the estimate of known prevalence in a finite population, according to the prevalence of moderate and severe malnutrition based on weight-for-height criteria, which was $5.3 \%$, according to the NNS-1988 [19]. The subsample required to determine the prevalence of anemia and the deficiencies of iron, zinc, and folic acid was also calculated with a formula to estimate population prevalence under the following assumptions: confidence level 95\%, maximum allowable error between the estimate and the parameter: 5\%; the resulting sample size was multiplied by a factor of 2.0 because of study design; a 35\% overestimate was calculated to account for potential hemolysis of the biological samples as well as for nonrespondents.

The only published information available at the time of the study related to micronutrient deficiency at country level was the prevalence of anemia found in NNS-99, which showed $48 \%$ prevalence for children between the ages of 12 and 23 months [20]; although not exactly on the same age range, this was the only available information to use. The resulting sample size was 1,030 children for each region. The sample size was weighted by the proportion with which each primary sampling unit contributed to the overall sample. The overall sample for RR was 4,120 children, and for IO, 3,090 children because an IO is not included in the Mexico City region.

\section{Data collection}

Data for the present study were collected between May 2000 and November 2001. In a series of structured interviews, we collected information on socioeconomic status; birth weight, size, and gestational age; complementary feeding; immunization status; morbidity on the month preceding the interview; nutritional anthropometric measures; and psychomotor development. Preliminary information has been published elsewhere [18]. Only parents who reported no infectious disease in their children in the two-week period preceding the interview were invited to participate in the micronutrient study. Those who agreed were asked to sign informed consents.

\section{Biological samples}

Three $\mathrm{ml}$ of venous blood were collected by droplets obtained from venipuncture by trained personnel. They were collected in 250-500 micro liter tubes filled with EDTA K2. Hemoglobin was determined by cyanide-metahemoglobin. Three amber-colored 800 micro-liter traceelement-free micro-tubes with separating gel were used to collect samples for determination of: ferritin by a two-tier immunoradiometric assay (CTK-IRMA, DiaSorin)-percentage of transferrin saturation and zinc by atomic mass 
spectrometry (AAnalyst 300, Perkin-Elmer, EUA). Four blood droplets were collected in filter paper to determine folic acid by a microbiological method using Lactobacillus casei [21]. Red blood cell analyses were carried out in each of the medical care unit laboratories. The blood samples obtained in the amber-colored micro-tubes were centrifuged for 20 minutes at $3000 \mathrm{rpm}$ and serum was separated. Samples for folic acid determination were saved in filter paper (Schleicher \& Schuell, No. 903), protected from light, and kept in dark envelopes with desiccant in plastic bags. Samples were kept frozen at $-20^{\circ} \mathrm{C}$ and sent for analysis at a Central facility where they were kept at $70^{\circ} \mathrm{C}$ until analyzed. All tests were run with the quality control samples recommended by the manufacturer. The standards set by the National Institute of Standards and Technology were used for the Fe, $\mathrm{Zn}$, and folic acid analyses: the coefficient of variation for serum Fe was $<5 \%$, for $\mathrm{Zn}<10 \%$, and for folic acid $<15 \%$. Determinations of ferritin, $\mathrm{Fe}$, and $\mathrm{Zn}$ were run against known values from control serum obtained from a pool of healthy donors with coefficients of variation $<10 \%$.

\section{Cut-offs}

Anemia: Hemoglobin values were adjusted for altitude with the following formula: $\% \mathrm{Hb}=[93.3197(10$ (0.0000251)(altitude) $)$ [ [22]. Cut-offs for hemoglobin (Hb) concentration to classify anemia were: $\mathrm{Hb}<13.5 \mathrm{~g} / \mathrm{dL}$ for children under 2 weeks of age; $\mathrm{Hb}<12.5 \mathrm{~g} / \mathrm{dL}$ for children between 2 and 3 weeks; $\mathrm{Hb}<10 \mathrm{~g} / \mathrm{dL}$ for children between 1 month and < than 2 months; $\mathrm{Hb}<9 \mathrm{~g} / \mathrm{dL}$ between 2 and $<3$ months; $\mathrm{Hb}<9.5$ between 3 and $<6$ months; $\mathrm{Hb}<10.5 \mathrm{~g} / \mathrm{dL}$ between 6 and $<12$ months; and $\mathrm{Hb}<10.7 \mathrm{~g} / \mathrm{dL}$ for children between 12 and 23 months $[23,24]$.

Iron deficiency: A cut-off of $<10 \mu \mathrm{g} / \mathrm{L}$ of ferritin was used [25].

Folic acid deficiency: Folic acid concentration $=57 \mathrm{ng} / \mathrm{mL}$ in whole blood [14].

Low zinc concentration: Serum zinc $<65 \mu \mathrm{g} / \mathrm{dL}[13,26]$.

\section{Statistical analyses}

Estimates of the prevalence of anemia, as well as deficiencies in iron, folic acid, and zinc, were carried out for the national and regional levels by age group, taking into consideration the sampling design. In other words, inferences for the whole population considered the stratified twostage design to apply expansion factors to the sampled population. Each point estimate is presented with $95 \%$ confidence intervals (CI). Statistical comparisons between regions and care regimens was carried out with Pearson's chi-square statistics, using the analyses routines for complex surveys; differences between means were assessed using linear models within the same routine to take into consideration the sampling design $[27,28]$. All statistical analyses were carried out with STATA version 8, special edition [29].

\section{Results}

Response rates were 64.2\% $(n=2,646)$ in RR and $74.7 \%$ $(n=2,309)$ in IO. Response rates differed in different regions of the country. For RR in the North region, we were able to recruit $76.2 \%$ of the expected sample; in the Center, 73.9\%; in the South, 58.7\%; and in Mexico City and periurban area, $44.8 \%$. For IO, the North recruited $85.8 \%$ of the expected sample; the Center, $93.6 \%$; and the South, $44.8 \%$. Children who participated in the micronutrient sample were significantly older than children who participated in the other parts of the larger study. In IO, there was also a significant difference in weight for age: children participating in the micronutrient sample had lower $\mathrm{Z}$ score $(-0.604)$ than those in the larger sample ($0.318)$. The other characteristics-including sex, height for age, years of education of either parent, and percent of working mothers' or fathers' occupation-showed no statistically significant differences between the micronutrient and the general sample (Table 1). Between regions, the only significant difference was found in the years of schooling of RR of mothers coming from the North, which were less than in the rest of the sample.

At the time of this survey, $84 \%$ of children in RR and $94 \%$ of children in IO had attended the health care facility searching for some kind of preventive service, like a wellbaby clinic or routine vaccination. Only $15.7 \%$ of children in RR and $6.4 \%$ in IO attended because of an acute illness. Additionally, 20\% of mothers reported that the child had had one or more signs of acute infection (e.g., diarrhea, cough, nasal discharge, fever) during the two weeks before the study, which was an exclusion criterion for the micronutrient sample, as acute infections affect the serum status of zinc and iron, making interpretation difficult.

There were some sociodemographic differences in the study sample when comparing regimen by region. Parents' schooling was higher in RR as compared with IO; a larger percentage of mothers in RR worked outside home (24.4\% vs. 8.2\%); and the proportion of houses with dirt floors was lower in RR than in IO (4.3\% vs. 33.1\%). Other differences included: at 4 months of age, $23.8 \%$ of children in RR were exclusively breast-fed, in comparison with $46.2 \%$ in IO; at this age, children in the South had a higher prevalence of exclusive breast feeding compared to the rest of the country ( $31.5 \%$ in RR and $48.4 \%$ in IO); the proportion of children still receiving some breast milk at 12 months of age was larger in the South than in the rest of the country: $26.9 \%$ in RR and $66.2 \%$ in IO. Lastly, the 
Table I: General characteristics of children studied for micronutrient deficiencies and children participating in the total survey

\begin{tabular}{|c|c|c|c|c|c|c|c|c|}
\hline & \multicolumn{4}{|c|}{ Regular Regimen (RR) } & \multicolumn{4}{|c|}{ IMSS-Oportunidades (IO) } \\
\hline & \multicolumn{2}{|c|}{ Micronutrient sample } & \multicolumn{2}{|c|}{ Survey sample } & \multicolumn{2}{|c|}{ Micronutrient sample } & \multicolumn{2}{|c|}{ Survey sample } \\
\hline & Mean & $95 \% \mathrm{Cl}$ & Mean & $95 \% \mathrm{Cl}$ & Mean & $95 \% \mathrm{Cl}$ & Mean & $95 \% \mathrm{Cl}$ \\
\hline Age (months) & $1 \mathrm{l} .4$ & $10.6,13.4$ & $8.6^{a}$ & $8.0,9.2$ & 13.4 & $12.3,14.6$ & $10.7^{a}$ & $12.3,14.6$ \\
\hline$<6(\%)$ & 13.2 & $8.5,19.7$ & 37.7 & $33.0,42.7$ & 4.9 & $2.8,8.2$ & 27.6 & $24.6,30.8$ \\
\hline $6-11$ & 38.2 & $34.8,41.7$ & 38.2 & $30.6,34.6$ & 31.0 & $24.0,39.1$ & 27.1 & $25.6,28.7$ \\
\hline $12-23$ & 48.7 & $41.8,55.6$ & 29.7 & $26.1,33.6$ & 64.1 & $57.4,70.2$ & 45.3 & $42.4,48.3$ \\
\hline Sex Male (\%) & 50.4 & $48.4,52.4$ & 51.5 & $50.6,52.4$ & 51.8 & $48.8,54.8$ & 51.2 & $49.5,52.9$ \\
\hline Weight for age (Z score) & -0.151 & $-0.308,0.005$ & 0.072 & $-0.04,0.188$ & -0.604 & $-0.615,-0.393$ & $-0.318^{b}$ & $-0.454,-0.186$ \\
\hline Height for age (Z score) & -0.188 & $-0.297,-0.078$ & -0.119 & $-0.278,0.039$ & -1.164 & $-1.585,-0.743$ & -0.873 & $-1.052,-0.694$ \\
\hline \multicolumn{9}{|c|}{ Characteristics of mothers } \\
\hline Years of study & 9.0 & $8.5,9.6$ & 9.2 & $8.8,9.5$ & 5.3 & $4.4,6.1$ & 5.3 & $4.8,5.7$ \\
\hline Work outside home (\%) & 24.4 & $18.4,31.5$ & 25.6 & $21.8,29.8$ & 8.2 & $6.5,10.3$ & 9.8 & $7.2,13.2$ \\
\hline \multicolumn{9}{|c|}{ Characteristics of fathers } \\
\hline Years of study & 9.2 & $8.7,9.7$ & 9.3 & $8.9,9.7$ & 5.3 & $4.5,6.2$ & 5.5 & $5.1,5.9$ \\
\hline \multicolumn{9}{|l|}{ Occupation (\%): } \\
\hline General services & 3.6 & $3.1,5.2$ & 4.0 & $2.3,5.6$ & 1.3 & $0.9,2.0$ & 1.2 & $0.9,1.6$ \\
\hline Manual laborer & 27.4 & $23.2,28.4$ & 25.7 & $23.1,32.1$ & 29.0 & $21.9,37.2$ & 28.6 & $21.2,37.4$ \\
\hline Clerk & 51.4 & $48.8,58.0$ & 53.4 & $43.0,59.8$ & 7.8 & $4.7,12.6$ & 7.3 & $4.6,11.6$ \\
\hline Small business owner & 4.6 & $4.2,5.8$ & 4.9 & $3.2,4.2$ & 4.4 & $2.8,6.9$ & 4.6 & $2.8,7.5$ \\
\hline Technician & 6.7 & $4.7,6.4$ & 5.5 & $5.3,8.3$ & 0.7 & $0.4,1.1$ & 0.6 & $0.3,1.0$ \\
\hline Professional & 5.2 & $3.7,5.6$ & 4.5 & $3.0,9.0$ & 2.1 & $1.4,3.1$ & 2.2 & $1.4,3.4$ \\
\hline Art and culture & 0.4 & $0.3,0.7$ & 0.5 & $0.2,1.0$ & 0.3 & $0.2,0.5$ & 0.3 & $0.1,0.5$ \\
\hline Land and agriculture & 0.7 & $0.8,2.5$ & 1.4 & $0.3,1.6$ & 52.9 & $43.6,62.0$ & 53.7 & $44.8,62.4$ \\
\hline Migrant & & & & & 1.3 & $0.7,2.6$ & 1.5 & $0.7,2.9$ \\
\hline
\end{tabular}

95\% Cl: $95 \%$ confidence interval

ap $<0.001{ }^{b} p=0.018$

prevalence of growth stunting in the country was $6.5 \%$ in $\mathrm{RR}$, in contrast with $22.4 \%$ in $\mathrm{IO}$, and growth stunting was more prevalent in the North for RR (7.4\%) and in the Center for IO (24.2\%).

In describing the sample included in this study, $13.2 \%$ of children in RR and $4.9 \%$ in $\mathrm{IO}$ were younger than 6 months of age; $38.2 \%$ and $31.0 \%$, respectively, were between 6 and 11 months old; and $48.7 \%$ and $64.1 \%$, respectively, were between 12 and 23 months of age. Sixty percent of children included in the sample had all four determinations for micronutrients, including $\mathrm{Hb}$, serum ferritin, serum zinc, and folic acid. The most common reason why the other $40 \%$ did not have complete lab determinations was because of insufficient blood sample. Only $3 \%$ of the samples were lipemic or hemolyzed, and thus unsuitable for analysis. Because of budgetary constraints, only half the samples were analyzed in the North and Center regions of IO; samples selected for analysis were drawn at random.

Results in Tables 2, 3, 4, 5, 6, 7 are shown by region and age group in each branch of IMSS. Resorting to the age groupings used in other published health surveys in the country, age categories included: less than 6 months, 611 months, and 12-23 months.

\section{Prevalence of anemia}

In RR, the prevalence of anemia for the whole country was $20.5 \%$, with significant differences between regions. The highest prevalence of anemia was found in the South $(37.4 \%)$ and in Mexico City and its periurban area (25.1\%) (Table 2) $(p=0.001)$. Prevalence of anemia at the national level was highest in children between 12 and 23 months of age $(22.7 \%)$ and lowest in children under 6 months of age (9.8\%); children between 6 and 11 months old showed $20.9 \%$ prevalence. In IO, prevalence of anemia at national level was $19.3 \%$, with no statistically significant differences in prevalence between regions (Table 2 ). There were no statistically significant differences in the prevalence of anemia between RR and IO $(p=0.635)$.

\section{Prevalence of iron deficiency}

The prevalence of iron deficiency, determined by ferritin concentration $<10 \mu \mathrm{g} / \mathrm{L}$, in RR was $32.6 \%$ (Table 3 ). Body iron stores were more depleted as age increased: in children younger than 6 months of age the prevalence was 6.8\%; for children between 6 and 11 months, it was 
Table 2: Hemoglobin concentration and prevalence of anemia, by region, age group, and care regimen

\begin{tabular}{|c|c|c|c|c|c|c|c|c|c|c|c|c|}
\hline \multirow[t]{3}{*}{ Region/Age groups } & \multicolumn{6}{|c|}{ Regular Regimen (RR) } & \multicolumn{6}{|c|}{ IMSS-Oportunidades (IO) } \\
\hline & \multirow[t]{2}{*}{$n$} & \multirow[t]{2}{*}{$N$} & \multicolumn{2}{|c|}{ Hemoglobin concentration $\mathrm{g} / \mathrm{dL}^{*}$} & \multicolumn{2}{|c|}{ Prevalence of anemia** } & \multirow[t]{2}{*}{$n$} & \multirow[t]{2}{*}{$N$} & \multicolumn{2}{|c|}{ Hemoglobin concentration $\mathrm{g} / \mathrm{dL}^{*}$} & \multicolumn{2}{|c|}{ Prevalence of anemia** } \\
\hline & & & Mean & $95 \% \mathrm{Cl}$ & $\%$ & $95 \% \mathrm{Cl}$ & & & Mean & $95 \% \mathrm{Cl}$ & $\%$ & $95 \% \mathrm{Cl}$ \\
\hline \multicolumn{13}{|l|}{ North } \\
\hline$<6$ months & 97 & 49416 & 11.5 & $10.9,12.0$ & 5.1 & $2.7,9.6$ & 142 & 992 & 11.7 & $11.3,12.1$ & 12.2 & $7.4,19.4$ \\
\hline $6-11$ months & 321 & 181422 & 11.5 & II.I, II.9 & 14.5 & $9.0,22.6$ & 223 & 1891 & 11.6 & $11.2,12.1$ & 18.0 & 7.1, 38.4 \\
\hline 12-23 months & 367 & 223234 & 11.7 & $11.5,11.9$ & 15.2 & $10.6,21.4$ & 519 & 5073 & 11.7 & $11.3,12.0$ & 21.3 & $8.0,45.9$ \\
\hline Total North & 785 & 454072 & 11.6 & $11.2,12.0$ & $13.8^{\mathrm{a}}$ & $9.8,19.2$ & 884 & 7956 & 11.7 & $11.3,12.0$ & $19.4^{\mathrm{a}}$ & $8.2,39.2$ \\
\hline \multicolumn{13}{|l|}{ Center } \\
\hline$<6$ months & 148 & 53494 & 11.4 & $10.9,11.8$ & 4.5 & $1.4,14.0$ & 79 & 2315 & 13.7 & $10.9,16.4$ & 11.2 & $5.8,20.4$ \\
\hline 6-11 months & 291 & 115119 & 11.7 & $11.4,12.0$ & 14.4 & $9.7,20.8$ & 273 & 6975 & 12.1 & $11.4,12.8$ & 17.8 & $6.3,41.1$ \\
\hline 12-23 months & 324 & 151064 & 11.7 & $11.1,12.2$ & 19.1 & $13.9,25.7$ & 612 & 16858 & 12.2 & $11.6,12.9$ & 18.3 & $10.7,29.5$ \\
\hline Total Center & 763 & 319677 & 11.7 & $11.2,12.2$ & $15.0^{a}$ & $10.0,21.8$ & 964 & 26148 & 12.3 & $11.8,12.9$ & $17.6^{\mathrm{a}}$ & $9.4,30.4$ \\
\hline \multicolumn{13}{|l|}{ South } \\
\hline$<6$ months & 27 & 14792 & 10.7 & $10.2,11.2$ & 31.3 & $20.6,44.5$ & 36 & 8701 & 11.3 & $10.9,11.8$ & 16.1 & $4.7,42.5$ \\
\hline 6-11 months & 227 & 78181 & 11.0 & $10.5,11.4$ & 36.1 & $20.0,56.1$ & 135 & 38100 & 11.6 & $11.4,11.9$ & 14.9 & $10.6,20.5$ \\
\hline Total South & 605 & 203035 & 11.0 & $10.5,11.5$ & $37.4^{\mathrm{a}}$ & $22.6,55.0$ & 461 & 124683 & 11.7 & $11.5,11.9$ & $19.7^{a}$ & $16.4,23.6$ \\
\hline \multicolumn{13}{|l|}{ Mexico City \& } \\
\hline$<6$ months & 67 & 32810 & 10.9 & $10.7,11.0$ & 15.6 & $13.2,18.3$ & & & & & & \\
\hline 6-11 months & 183 & 111755 & 11.2 & $11.0,11.5$ & 27.3 & $21.0,34.6$ & & & & & & \\
\hline $12-23$ months & 245 & $|4056|$ & 11.4 & $11.2,11.6$ & 25.5 & $22.2,29.1$ & & & & & & \\
\hline Total Mexico City \& & 495 & 285126 & 11.3 & $11.0,11.5$ & $25 . I^{\mathrm{a}}$ & $21.2,29.3$ & & & & & & \\
\hline \multicolumn{13}{|l|}{ NATIONAL } \\
\hline$<6$ months & 339 & 150512 & 11.2 & $11.0,11.4$ & 9.8 & $6.3,14.8$ & 257 & 12008 & 11.8 & $11.0,12.6$ & 14.8 & $5.6,33.6$ \\
\hline 6-1I months & 1022 & 486477 & 11.4 & $11.2,11.6$ & 20.9 & $17.0,25.4$ & 631 & 46966 & 11.7 & $11.4,11.9$ & 15.5 & $11.2,21.0$ \\
\hline I2-23 months & 1287 & 624921 & 11.5 & $11.4,11.7$ & 22.7 & $19.2,26.6$ & $|42|$ & 99813 & 11.8 & $11.6,12.1$ & 21.7 & $17.3,27.0$ \\
\hline TOTAL NATIONAL & 2648 & 1261910 & 11.4 & $11.3,11.6$ & $20.5^{b}$ & $17.3,24.1$ & 2309 & 158787 & 11.8 & $11.6,12.0$ & $19.3^{\mathrm{b}}$ & $16.1,23.0$ \\
\hline
\end{tabular}

$N$ Population size (expanded sample)

* Hemoglobin concentration adjusted by altitude.

** Hemoglobin $(\mathrm{Hb})<13.5 \mathrm{~g} / \mathrm{dL}$ for children under 2 weeks of age, $\mathrm{Hb}<12.5 \mathrm{~g} / \mathrm{dL}$ for children between 2 and 3 weeks of age, $\mathrm{Hb}<10 \mathrm{~g} / \mathrm{dL}$ for children between $\mathrm{I}$ and $2 \mathrm{months}$ old, $\mathrm{Hb}<$ 9.0 for children between 2 and 3 months old $\mathrm{Hb}<9.5$ for children between 3 and 6 months old, $\mathrm{Hb}<10.5 \mathrm{~g} / \mathrm{dL}$ for children between 6 and $<12$ months old and $\mathrm{Hb}<10.7 \mathrm{~g} / \mathrm{dL}$ for children between 12 and 23 months old.

\& Mexico City and periurban area

a Comparing between regions, $\mathrm{RR}=0.00 \mathrm{I} ; \mathrm{IO}=0.908$

$\mathrm{b}$ Comparing between regimens, national level, $p=0.635$ 
Table 3: Ferritin concentration and prevalence of iron deficiency, by region, age group, and care regimen

Region/Age groups Regular Regimen (RR) IMSS-Oportunidades (IO)

\begin{tabular}{llll}
\hline$n$ & $N$ & Ferritin concentration $\mu \mathrm{g} / \mathrm{L}$ & Prevalence of iron deficiency*
\end{tabular} Mean $\quad 95 \% \mathrm{Cl} \quad \% \quad 95 \% \mathrm{Cl}$ n $\quad N$

Ferritin concentration $\mu \mathrm{g} / \mathrm{L}$ Prevalence of iron deficiency* Mean $\quad 95 \% \mathrm{Cl}$ $\%$ $95 \% \mathrm{Cl}$

North

$<6$ months

6-11 months

12-23 months

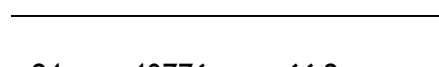

Total North

$84 \quad 40776 \quad 66$

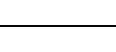

(2)

72

$\begin{array}{llll}268 & 138802 & 30.6 & 25.2,35.0\end{array}$

$\begin{array}{cc}4.3 & 2.6,7.1 \\ 25.6 & 18.6,34.2 \\ 44.1 & 26.5,63.3\end{array}$

$72 \quad 460$

(1)

(2)

$295 \quad 163476$

$\begin{array}{lll}647 & 343054 & 27.9\end{array}$

I 5.3, 40.5

$31.9^{a}$

$23.5,41.6$

$139 \quad 1148$

132.3
26.4

$64.1,200.5$
$16.6,36.2$

6.1
36.9

$2.3,15.2$

$\begin{array}{lll}1332 & 3239 & 28.0\end{array}$

$15.2,40.8$

43.3

$30.1,44.2$

$\begin{array}{lll}543 & 4848 & 37.5\end{array}$

23.7, 51.4

$38.2^{a}$

$32.6,54.6$

\section{Center}

$<6$ months

6-1I months

12-23 months

$\begin{array}{llll}147 & 55228 & 96.4 & 56.2,136.6\end{array}$

$4.6 \quad 1.5,13.0$

$\begin{array}{lll}33 & 703 & 65.6\end{array}$

$16.2,23.0$

19.4

13.4, 23.0

39.0
$26.8^{\text {a }}$

22.7, 31.4

$\begin{array}{lll}138 & 3644 & 21.1\end{array}$

$8.5,122.7$

$4.6,37.5$

I.0, 58.3

I.0, 54. I

13.8

49.7

South

$<6$ months

6-11 months

12-23 months

$777 \quad 352264$

34.5

$22.0,47.0$

$493 \quad 13615 \quad 26.6$

48.1
46.7

$8.1,22.4$

$30.4,69.1$

$\begin{array}{lll}65.8,97.9 & 21.0 & 13.5,31.3\end{array}$

I5.4, $23.4 \quad 37.3$

$30.4,44.8$

$19 \quad 5007 \quad 50.4$

$\begin{array}{ccc}97 & 25128 & 59.3 \\ 207 & 54992 & 35.8\end{array}$

$33.1,67.8$

24.0, 94.6

$16.7,54.9$

I8.6, 68.6

21.8
24.4
24.3

$37.5,58.8$

Total South

$\begin{array}{lll}\mathbf{6 6 5} & 217622 & \mathbf{2 0 . 2}\end{array}$

12.0, $27.9 \quad 46.2^{\mathrm{a}}$

37.4, 55.3

$\begin{array}{lll}323 & 85127 & 43.6\end{array}$

$4.6,11.2$

$<6$ months

6-1I months

$\begin{array}{lll}64 & 30872 & 87.9\end{array}$

$70.5,105.2$

7.2

12-23 months

$\begin{array}{lll}152 & 77977 & 32.2\end{array}$

28.9, 35.6

28.8

$29.5,44.4$

Total Mexico City \&

$\begin{array}{lll}195 & 88543 & 26.4 \\ 411 & 197392 & 38.3\end{array}$

16.2, 36.5

36.7

| 9. I, 4 I.

\section{NATIONAL}

6-1I months

$\begin{array}{lll}320 & |4| 228 \quad 84.3\end{array}$

6.8

$63.2,105.5$

23.9, 33.3

I5.I, 20.4

6.8
26.7
43.6

$4.3,10.9$

24.2, 36.4

$32.6^{\mathrm{b}}$

$22.8,30.9$

$37.1,50.2$

$\begin{array}{ccc}124 & 6170 & 58.3 \\ 374 & 29920 & 53.4 \\ 861 & 67500 & 34.0 \\ 1359 & 100230 & \mathbf{4 1 . 1}\end{array}$

4I.2, 75.4

$21.7,85.1$

$17.9,50.2$

19.7

28.0

II.4, 31.8

28.8, 36.6

20.0, 62. I

$27.8^{\mathrm{b}}$

13.2, 49.7

$22.3,35.5$

20.2, 36.9

$N$ Population size (expanded sample)

* Ferritin $<10 \mu \mathrm{g} / \mathrm{L}$

\& Mexico City and periurban area

\& Mexico City and periurban area

b Comparing between regimens, national level, $p=0.287$ 
Table 4: Iron nutritional status by hemoglobin and ferritin concentrations, by region and care regimen

\begin{tabular}{|c|c|c|c|c|c|c|c|c|c|c|c|c|c|c|c|c|c|c|c|c|}
\hline \multirow[b]{3}{*}{ Iron status } & \multicolumn{20}{|c|}{ Regular Regimen (RR) } \\
\hline & \multicolumn{4}{|c|}{$\begin{array}{c}\text { Total Regular Regimen } \\
n=2324 \\
N=1022247\end{array}$} & \multicolumn{4}{|c|}{$\begin{array}{c}\text { North } \\
n=639 \\
N=337,221\end{array}$} & \multicolumn{4}{|c|}{$\begin{array}{c}\text { Center } \\
n=712 \\
N=301,192\end{array}$} & \multicolumn{4}{|c|}{$\begin{array}{c}\text { South } \\
n=574 \\
N=193,493\end{array}$} & \multicolumn{4}{|c|}{$\begin{array}{l}\text { Mexico City and periurban area } \\
\qquad \begin{array}{c}n=399 \\
N=190,340\end{array}\end{array}$} \\
\hline & $n$ & $\mathbf{N}$ & $\%$ & $95 \% \mathrm{Cl}$ & $n$ & $\mathbf{N}$ & $\%$ & $95 \% \mathrm{Cl}$ & $n$ & $N$ & $\%$ & $95 \% \mathrm{Cl}$ & $n$ & $N$ & $\%$ & $95 \% \mathrm{Cl}$ & $n$ & $\mathbf{N}$ & $\%$ & $95 \% \mathrm{Cl}$ \\
\hline Normal a & 1298 & 582313 & $57.0^{f}$ & $52.1,61.7$ & 375 & 206383 & $61.2 \mathrm{~g}$ & $50.3,71.1$ & 499 & 203988 & $67.7 \mathrm{~g}$ & $58.9,75.4$ & 207 & 67839 & $35.1 \mathrm{~g}$ & $32.4,37.8$ & 217 & 104103 & 54.78 & $41.9,66.9$ \\
\hline Iron deficiency without anemia b & 530 & 224550 & 22.0 & $18.2,26.3$ & 168 & 82056 & 24.3 & $17.8,32.3$ & 116 & 51229 & 17.0 & $14.6,19.8$ & 169 & 53701 & 27.8 & $16.0,43.6$ & 77 & 37563 & 19.7 & 14.9, 25.7 \\
\hline Anemiac $^{c}$ & 496 & 215385 & 21.0 & I7.I, 25.6 & 96 & 48781 & 14.5 & $11.0,18.8$ & 97 & 45976 & 15.3 & $10.1,22.5$ & 198 & 71953 & 37.2 & $22.7,54.5$ & 105 & 48675 & 25.6 & $18.6,34.0$ \\
\hline Iron deficiency anemia ${ }^{d}$ & 248 & 105942 & 10.3 & $8.6,12.4$ & 49 & 24073 & 7.1 & $4.6,10.9$ & 59 & 30173 & 10.0 & $7.0,14.1$ & 102 & 34511 & 17.8 & I2.2, 25.4 & 38 & 17185 & 9.0 & $5.2,15.3$ \\
\hline Anemia due to other causes e & 248 & 109443 & 10.7 & $8.0,14.2$ & 47 & 24708 & 7.3 & $4.8,11.1$ & 38 & 15803 & 5.2 & $2.5,10.5$ & 96 & 37442 & 19.4 & $10.2,33.7$ & 67 & 31490 & 16.5 & $13.0,20.8$ \\
\hline
\end{tabular}

\begin{tabular}{|c|c|c|c|c|c|c|c|c|c|c|c|c|c|c|c|c|}
\hline \multicolumn{17}{|c|}{ IMSS-Oportunidades (IO) } \\
\hline \multirow[b]{2}{*}{ Iron status } & \multicolumn{4}{|c|}{$\begin{array}{c}\text { Total IMSS-Oportunidades } \\
\qquad \begin{array}{c}n=1325 \\
N=98150\end{array}\end{array}$} & \multirow[b]{2}{*}{$n$} & \multicolumn{3}{|c|}{$\begin{array}{c}\text { North } \\
n=539 \\
N=4195\end{array}$} & \multicolumn{4}{|c|}{$\begin{array}{c}\text { Center } \\
n=479 \\
N=10882\end{array}$} & \multicolumn{4}{|c|}{$\begin{array}{c}\text { South } \\
n=307 \\
N=83074\end{array}$} \\
\hline & $n$ & $N$ & $\%$ & $95 \% \mathrm{Cl}$ & & $\mathbf{N}$ & $\%$ & $95 \% \mathrm{Cl}$ & $n$ & $\mathbf{N}$ & $\%$ & $95 \% \mathrm{Cl}$ & $n$ & $N$ & $\%$ & $95 \% \mathrm{Cl}$ \\
\hline Normal a & 681 & 60873 & $62.0^{f}$ & $53.2,70.2$ & 294 & 2308 & $55.0^{\mathrm{h}}$ & $46.8,63.0$ & 192 & 4668 & $42.9^{\mathrm{h}}$ & $33.9,52.4$ & 195 & 53897 & $64.9^{\mathrm{h}}$ & $54.6,73.9$ \\
\hline Iron deficiency without anemia $b$ & 362 & 20110 & 20.5 & 13.8, 29.2 & 132 & 1140 & 27.2 & $21.0,34.4$ & 171 & 4240 & 39.0 & $29.3,49.6$ & 59 & 14730 & 17.7 & 10.9, 27.6 \\
\hline Anemiac & 282 & 17167 & 17.5 & $13.2,22.8$ & 113 & 746 & 17.8 & $9.1,31.9$ & 116 & 1974 & 18.1 & $8.0,36.2$ & 53 & 14447 & 17.4 & $12.7,23.4$ \\
\hline Iron deficiency anemia $\mathrm{d}$ & 137 & 6873 & 7.0 & $4.7,10.3$ & 62 & 441 & 10.5 & $4.8,21.4$ & 57 & 984 & 9.0 & $4.2,18.3$ & 18 & 5448 & 6.6 & $4.0,10.5$ \\
\hline Anemia due to other causes $\mathrm{e}$ & 145 & 10294 & 10.5 & $7.4,14.7$ & 51 & 305 & 7.3 & $3.9,13.2$ & 59 & 990 & 9.1 & $3.6,21.1$ & 35 & 5448 & 10.8 & $7.4,15.7$ \\
\hline
\end{tabular}

N Population size (expanded sample)

10. a Hemoglobin above the cut-off point for anemia and ferritin $>=10 \mu \mathrm{g} / \mathrm{L}$

\&े ${ }^{b}$ Ferritin $<10 \mu \mathrm{g} / \mathrm{L}$ and $\mathrm{Hb}>=$ cut-off for anemia

$\stackrel{c}{\sim} \quad \mathrm{Hb}<$ cut-off for anemia

- $d$ Ferritin $<10 \mu \mathrm{g} / \mathrm{L}$ and $\mathrm{Hb}<$ cut-off for anemia

e Ferritin $=10 \mu \mathrm{g} / \mathrm{L}$ and $\mathrm{Hb}<$ cut-off for anemia

Comparing nutritional iron status between regimens $p=0.499$

g,h Comparing iron status between regions $8 \mathrm{RR}<0.00 \mathrm{I}$; $\mathrm{h} 1 \mathrm{O}=0.012$ 
Table 5: Folic acid concentration and prevalence of folic acid deficiency, by region, age group, and care regimen

\begin{tabular}{|c|c|c|c|c|c|c|c|c|c|c|c|c|}
\hline \multirow[t]{3}{*}{ Region/Age groups } & \multicolumn{6}{|c|}{ Regular Regimen (RR) } & \multicolumn{6}{|c|}{ IMSS-Oportunidades (IO) } \\
\hline & \multirow[t]{2}{*}{$n$} & \multirow[t]{2}{*}{$N$} & \multicolumn{2}{|c|}{ Serum folic acid concentration $\mathrm{ng} / \mathrm{mL}$} & \multicolumn{2}{|c|}{ Prevalence of folic acid deficiency* } & \multirow[t]{2}{*}{$n$} & \multirow[t]{2}{*}{$N$} & \multicolumn{2}{|c|}{ Serum folic acid concentration $\mathrm{ng} / \mathrm{mL}$} & \multicolumn{2}{|c|}{ Prevalence of folic acid deficiency* } \\
\hline & & & Mean & $95 \% \mathrm{Cl}$ & $\%$ & $95 \% \mathrm{Cl}$ & & & Mean & $95 \% \mathrm{Cl}$ & $\%$ & $95 \% \mathrm{Cl}$ \\
\hline \multicolumn{13}{|l|}{ North } \\
\hline$<6$ months & 96 & 48932 & 102.7 & $51.0,154.3$ & 25.2 & II.0, 47.9 & 97 & 601 & 124.8 & 105.0, 144.6 & 6.6 & $2.5,16.3$ \\
\hline $6-11$ months & 298 & 176084 & 120.9 & $65.4,176.3$ & 14.5 & $3.2,47.1$ & 154 & 1169 & 120.5 & $98.6,142.4$ & 6.9 & $2.4,18.3$ \\
\hline $12-23$ months & 314 & 196452 & 112.1 & $72.0,152.2$ & 13.4 & $2.7,46.5$ & 362 & 2640 & 125.8 & $110.8,140.9$ & 3.1 & $1.4,6.5$ \\
\hline Total North & 708 & 421468 & 114.7 & $55.9,173.4$ & $15.2^{\mathrm{a}}$ & $3.3,48.7$ & 613 & 4410 & 124.3 & $109.2,139.4$ & $4.6^{a}$ & $2.3,9.0$ \\
\hline \multicolumn{13}{|l|}{ Center } \\
\hline$<6$ months & 142 & 49708 & 131.4 & $100.6,162.2$ & 9.7 & $2.2,33.5$ & 72 & 2065 & 163.6 & $149.4,177.8$ & 0.0 & ... \\
\hline $6-11$ months & 291 & 119236 & 149.0 & 122.1, 175.9 & 4.3 & $1.4,13.0$ & 239 & 6278 & 168.1 & $142.3,193.9$ & 3.0 & $0.8,11.0$ \\
\hline $12-23$ months & 333 & 159516 & 127.5 & $99.6,155.4$ & 6.4 & $1.7,21.7$ & 546 & 15902 & 147.1 & $\mid 13.6,180.7$ & 1.4 & $0.2,7.3$ \\
\hline Total Center & 766 & 328460 & 135.9 & $103.8,167.9$ & $6.1^{a}$ & $1.7,20.2$ & 857 & 24246 & 154.0 & $124.9,183.0$ & $1.7^{\mathrm{a}}$ & $0.4,6.2$ \\
\hline \multicolumn{13}{|l|}{ South } \\
\hline$<6$ months & 10 & 3973 & 118.7 & $58.3,179.2$ & 16.6 & $3.6,51.7$ & 25 & 6861 & 155.9 & |35.1, 176.7 & 0.0 & -.- \\
\hline $6-11$ months & 202 & 62292 & 111.7 & $81.0,142.4$ & 8.6 & $1.6,36.1$ & 110 & 34142 & 134.4 & $108.5,160.3$ & 5.8 & $2.4,13.3$ \\
\hline 12-23 months & 320 & 85651 & 111.4 & $88.4,134.3$ & 14.1 & $4.5,36.1$ & 228 & 68987 & 112.0 & $81.9,142.1$ & 12.6 & $3.0,40.4$ \\
\hline Total South & 532 & 151917 & 111.7 & $82.8,140.5$ & $11.9^{\mathrm{a}}$ & $3.4,34.2$ & 363 & 109991 & 121.7 & $92.9,150.5$ & $9.7^{\mathrm{a}}$ & $2.5,30.6$ \\
\hline \multicolumn{13}{|l|}{ Mexico City\& } \\
\hline$<6$ months & 45 & 23968 & 107.0 & $103.6,110.3$ & 5.4 & $4.1,7.2$ & & & & & & \\
\hline $6-11$ months & 118 & 63812 & 121.3 & $108.7,133.9$ & 5.0 & $2.2,11.0$ & & & & & & \\
\hline 12-23 months & 137 & 70367 & 106.8 & $104.2,109.5$ & 5.6 & $0.7,32.1$ & & & & & & \\
\hline Total Mexico City \& & 300 & 158146 & 112.7 & $105.0,120.3$ & $5.1^{\mathrm{a}}$ & $2.6,10.1$ & & & & & & \\
\hline \multicolumn{13}{|l|}{ NATIONAL } \\
\hline$<6$ months & 293 & $12658 \mid$ & 115.3 & $87.3,143.2$ & 15.1 & $5.8,33.7$ & 194 & 9528 & 155.6 & 139.9, 171.3 & 0.4 & $0.1,1.4$ \\
\hline $6-11$ months & 909 & 421424 & 127.5 & $101.9,153.2$ & 9.3 & $3.2,24.2$ & 503 & 41589 & 139.1 & $119.7,158.5$ & 5.4 & $2.6,11.0$ \\
\hline $12-23$ months & 1104 & 511986 & 116.0 & $98.0,134.1$ & 10.3 & $4.3,22.5$ & 1136 & 87530 & 118.8 & $95.2,142.4$ & 10.2 & $2.7,32.0$ \\
\hline TOTAL NATIONAL & 2306 & $105999 \mid$ & 120.5 & $99.3,141.7$ & $10.4^{\mathrm{b}}$ & $4.0,24.4$ & 1833 & 138646 & 127.4 & $105.5,149.4$ & $8.1^{\mathrm{b}}$ & $2.4,23.7$ \\
\hline
\end{tabular}

$N$ Population size (expanded sample)

$* \leq 57 \mathrm{ng} / \mathrm{mL}$ in whole blood sample

\& Mexico City and periurban area

a Comparing between regions within each regimen, $\mathrm{RR}=0.458 ; \mathrm{IO}=0.046$.

b Comparing between regimens, national level, $p=0.720$ 
Table 6: Zinc concentration and prevalence of low zinc concentration, by region, age group, and care regimen

\begin{tabular}{|c|c|c|c|c|c|c|c|c|c|c|c|c|}
\hline \multirow[b]{3}{*}{ Region/Age Groups } & \multicolumn{6}{|c|}{ Regular Regimen (RR) } & \multicolumn{6}{|c|}{ IMSS-Oportunidades (IO) } \\
\hline & \multirow[b]{2}{*}{$n$} & \multirow[b]{2}{*}{$\mathrm{N}$} & \multicolumn{2}{|c|}{ Zinc concentration $\mu / \mathrm{dL}$} & \multicolumn{2}{|c|}{ Prevalence of low zinc concentration* } & \multirow[b]{2}{*}{$n$} & \multirow[b]{2}{*}{$N$} & \multicolumn{2}{|c|}{ Zinc concentration $\mu / d \mathrm{~L}$} & \multicolumn{2}{|c|}{ Prevalence of low zinc concentration* } \\
\hline & & & Mean & $95 \% \mathrm{Cl}$ & $\%$ & $95 \% \mathrm{Cl}$ & & & Mean & $95 \% \mathrm{Cl}$ & $\%$ & $95 \% \mathrm{Cl}$ \\
\hline \multicolumn{13}{|l|}{ North } \\
\hline$<6$ months & 94 & 46028 & 82.8 & $76.6,89.0$ & 20.6 & $8.1,43.3$ & 60 & 406 & 83.1 & $61.3,104.9$ & 35.2 & $11.0,70.5$ \\
\hline $6-11$ months & 289 & 151419 & 86.4 & $74.0,98.8$ & 22.7 & $10.2,43.2$ & 126 & 1104 & 84.0 & $70.7,97.3$ & 21.6 & $6.3,53.1$ \\
\hline 12-23 months & 330 & 189064 & 81.6 & $66.3,96.9$ & 31.2 & $14.6,54.5$ & 307 & 2914 & 83.0 & $78.5,87.6$ & 21.1 & $8.8,42.7$ \\
\hline Total North & 713 & 386511 & 83.6 & $70.8,96.4$ & $26.6^{\mathrm{a}}$ & II.6, 50.1 & 493 & 4425 & 83.3 & $75.5,91.0$ & $22.5^{a}$ & 8.2, 48.7 \\
\hline \multicolumn{13}{|l|}{ Center } \\
\hline$<6$ months & 148 & 55798 & 76.3 & $70.5,82.1$ & 34.6 & $20.3,52.2$ & 33 & 981 & 72.5 & $49.9,95.1$ & 63.2 & $30.1,87.2$ \\
\hline $6-11$ months & 293 & 123937 & 76.1 & $68.9,83.2$ & 31.3 & $23.1,41.0$ & $|3|$ & 3516 & 79.8 & $73.2,86.5$ & 29.4 & $21.4,38.8$ \\
\hline 12-23 months & 351 & 178793 & 75.1 & $73.3,76.9$ & 32.3 & $25.6,39.8$ & 315 & 9230 & 79.7 & $74.3,85.1$ & 26.6 & $19.2,35.6$ \\
\hline Total Center & 792 & 339398 & 75.6 & $71.7,79.6$ & $32.4^{\mathrm{a}}$ & $26.0,39.4$ & 479 & 13727 & 79.2 & $75.4,83.0$ & $29.9^{a}$ & $24.1,36.5$ \\
\hline \multicolumn{13}{|l|}{ South } \\
\hline$<6$ months & 29 & 15312 & 72.4 & $64.8,79.9$ & 38.1 & $21.2,58.5$ & 25 & 6605 & 87.5 & $74.9,100.2$ & 5.9 & $1.4,22.1$ \\
\hline $6-11$ months & 247 & 81878 & 77.7 & $72.7,82.7$ & 25.6 & $17.3,36.2$ & 100 & 25968 & 91.6 & $84.7,98.5$ & 14.4 & $6.6,28.8$ \\
\hline 12-23 months & 404 & 124130 & 78.6 & $71.6,85.7$ & 26.1 & $18.4,35.6$ & 211 & 55166 & 90.4 & $82.9,97.9$ & 9.0 & $3.2,22.8$ \\
\hline Total South & 680 & 222491 & 77.9 & $71.8,83.9$ & $26.6^{\mathrm{a}}$ & $18.4,36.8$ & 336 & 87740 & 90.5 & $84.3,96.8$ & $10.4^{a}$ & $5.2,19.5$ \\
\hline \multicolumn{13}{|l|}{ Mexico City and periurban area } \\
\hline$<6$ months & 60 & 28968 & 87.5 & $70.4,104.5$ & 20.2 & $4.0,60.6$ & & & & & & \\
\hline $6-11$ months & 161 & 82599 & 81.6 & $65.2,98.0$ & 25.6 & 19.0, 33.6 & & & & & & \\
\hline 12-23 months & 198 & 89905 & 88.2 & $73.0,103.3$ & 22.5 & $11.8,38.8$ & & & & & & \\
\hline Total Mexico City and periurban area & 419 & 211391 & 85.4 & $69.1,101.7$ & $24.0^{\mathrm{a}}$ & $12.8,40.4$ & & & & & & \\
\hline \multicolumn{13}{|l|}{ NATIONAL } \\
\hline$<6$ months & 331 & 146106 & 80.1 & $74.3,85.9$ & 27.7 & $16.6,42.4$ & 118 & 7992 & 805 & $74.6,96.3$ & 14.4 & $6.7,28.3$ \\
\hline $6-11$ months & 990 & 439833 & 81.0 & $74.9,87.1$ & 26.2 & $19.6,34.2$ & 357 & 30588 & 90.0 & $83.7,96.2$ & 16.4 & $9.0,27.8$ \\
\hline 12-23 months & 1283 & 581893 & 80.0 & $74.4,85.6$ & 29.1 & $22.2,37.1$ & 833 & 67311 & 88.6 & $82.4,94.9$ & 12.0 & $6.2,21.7$ \\
\hline TOTAL NATIONAL & 2604 & |17979| & 80.4 & $74.9,85.8$ & $27.9 \mathrm{~b}$ & $20.9,36.1$ & 1332 & 108912 & $\mathbf{8 8 . 8}$ & $83.6,94.0$ & 13.4b & $8.3,21.0$ \\
\hline
\end{tabular}

$N$ Population size (expanded sample)

* Zinc concentration $<65 \mu / \mathrm{dL}$

a Comparing between regions within each regimen, $R R=0.746 ; 1 O=0.003$.

b Comparing between regimens, national level, $p=0.005$ 
Table 7: Folic acid deficiency and low zinc concentration by iron status

\begin{tabular}{|c|c|c|c|c|c|c|c|c|}
\hline \multirow{3}{*}{ Iron status } & \multicolumn{8}{|c|}{ Regular Regimen (RR) } \\
\hline & \multicolumn{4}{|c|}{$\begin{array}{l}\text { Folic acid deficiency by iron status } h \\
\qquad \begin{array}{l}n=2025 \\
N=856,699\end{array}\end{array}$} & \multicolumn{4}{|c|}{$\begin{array}{l}\text { Low zinc concentration by iron status } \\
\qquad \begin{array}{c}i \\
N=2268 \\
N=996,996\end{array}\end{array}$} \\
\hline & Cases/n & $\mathbf{N}$ & $\%$ f & $95 \% \mathrm{Cl}$ & Cases/n & $\mathbf{N}$ & $\% g$ & $95 \% \mathrm{Cl}$ \\
\hline Normala & $91 / 1135$ & 493903 & 8.3 & $2.5,24.1$ & $328 / 1264$ & 567943 & 26.1 & $19.4,34.1$ \\
\hline Iron deficiency without anemiab & $43 / 474$ & 194059 & 10.8 & $3.8,26.9$ & $130 / 515$ & 216433 & 25.8 & $18.4,34.9$ \\
\hline Anemiac & $80 / 416$ & 168736 & 19.3 & $10.5,32.8$ & $139 / 489$ & 212621 & 30.2 & $22.1,39.3$ \\
\hline Iron deficiency anemiad & $39 / 2 / 4$ & 84405 & 18.5 & $10.9,29.7$ & $76 / 246$ & 105359 & 32.7 & $23.0,44.3$ \\
\hline Anemia due to other causes e & $4 / / 202$ & 84331 & 20.1 & $8.9,39.5$ & $63 / 243$ & 107262 & 27.8 & $18.3,39.8$ \\
\hline \multirow{3}{*}{ Iron status } & \multicolumn{5}{|c|}{ IMSS-Oportunidades (IO) } & & & \\
\hline & \multicolumn{4}{|c|}{$\begin{array}{l}\text { Folic acid deficiency by iron statush } \\
\qquad \begin{array}{l}n=1062 \\
N=88,848\end{array}\end{array}$} & \multicolumn{4}{|c|}{$\begin{array}{l}\text { Low zinc concentration by iron status } \\
\qquad \begin{array}{l}n=1214 \\
N=92,310\end{array}\end{array}$} \\
\hline & Cases/n & $\mathbf{N}$ & $\%$ f & $95 \% \mathrm{Cl}$ & Cases/n & $\mathbf{N}$ & $\% g$ & $95 \% \mathrm{Cl}$ \\
\hline Normala & $27 / 517$ & 55564 & 8.8 & $2.6,25.5$ & $128 / 616$ & 56805 & $\mathrm{II} .4$ & $5.3,22.8$ \\
\hline Iron deficiency without anemiab & $8 / 298$ & 18733 & 8.3 & $1.5,34.5$ & $80 / 334$ & 19425 & 9.9 & $6.2,15.4$ \\
\hline Anemiac & $18 / 247$ & 14550 & 12.8 & $2.4,46.8$ & $85 / 264$ & 16080 & 15.1 & $7.9,26.8$ \\
\hline Iron deficiency anemia ${ }^{d}$ & $1 / / / 22$ & $6 / 43$ & 23.8 & $6.0,60.5$ & $39 / 128$ & 6753 & 8.2 & $3.8,16.7$ \\
\hline Anemia due to other causes ${ }^{\mathrm{e}}$ & $7 / / 25$ & 8407 & 4.8 & $0.3,45.8$ & $46 / 136$ & 9327 & 20.1 & $8.8,39.5$ \\
\hline
\end{tabular}

N Population size (expanded sample)

a Hemoglobin above the cut-off point for anemia and ferritin $>=10 \mu \mathrm{g} / \mathrm{L}$

b Ferritin $<10 \mu \mathrm{g} / \mathrm{L}$ and $\mathrm{Hb}>=$ cut-off for anemia

c $\mathrm{Hb}<$ cut-off for anemia

d Ferritin $<10 \mu \mathrm{g} / \mathrm{L}$ and $\mathrm{Hb}<$ cut-off for anemia

e Ferritin $\geq 10 \mu \mathrm{g} / \mathrm{L}$ and $\mathrm{Hb}<$ cut-off for anemia

f Comparing folic acid deficiency between iron status, $R R=0.102 ; 1 O p=0.208$

g Comparing low zinc concentration between iron status, $\operatorname{RR} p=0.444 ; \mathrm{IO} p=0.238$

h Comparing folic acid deficiency between RR and IO according to iron status $(p=0.387)$

i Comparing low zinc concentration between RR and IO according to iron status $(p=0.220)$

26.7\%; and for children $12-23$ months old, it was $43.6 \%$. There were marked differences in overall prevalence between regions, with the lower and higher values in the Center $(26.8 \%)$ and South $(46.2 \%)$, respectively $(p=$ 0.015) (Table 3).

In IO, iron deficiency had a prevalence of $27.8 \%$. As described for RR, iron deficiency increased with age: in children younger than 6 months of age, prevalence of iron deficiency was 19.7\%; in children between 6 and 11 months old, prevalence was $28.0 \%$; and in children $12-$ 23 months old, prevalence of iron deficiency was $28.4 \%$. The highest prevalence of iron deficiency was found in the Center $(46.7 \%)$, followed by the North $(38.2 \%)$ and by the South $(24.2 \%)(p=0.007)$. There were no statistically significant differences in the overall prevalence of iron deficiency between regimens $(p=0.287)$.
Other studies have used a cut-off for ferritin concentration $<12 \mu \mathrm{g} / \mathrm{L}$ to evaluate low iron stores in this age group, so we ran a second analysis with this cut-off. In RR, the prevalence of iron deficiency at the national level was $37.0 \%$ (95\% CI: 33.6, 40.6), and by regions, the North showed $36.3 \%$ (95\% CI: 28.1, 45.4), the Center, 32.4\% (95\% CI: $28.4,36.7)$, the South, $50.8 \%$ (95\% CI: $42.5,59.2)$, and Mexico City and periurban area, 31.5\% (95\% CI: 23.0 , 41.4). In IO, the national prevalence was $32.1 \%$ (95\% CI: 23.7, 41.9); in the North, $42.4 \%$ (95\% CI: $37.5,47.4)$; in the Center, $50.2 \%$ (95\% CI: 35.5, 64.9); and in the South, 28.7\% (95\% CI: 19.6, 39.9). Between regions there were statistically significant differences, with a $p=0.006$ for RR and $p=0.017$ for IO, but the prevalence of iron deficiency between the two IMSS regimens was not statistically significantly different $(p=0.326)$. 


\section{Iron status by hemoglobin and ferritin concentration}

In RR, $57.0 \%$ of all children had a normal iron status, as judged by normal hemoglobin and ferritin concentrations. Thus, $43 \%$ of children had iron deficiency and/or anemia. Only half $(10.3 \%)$ of the $21 \%$ of cases with anemia were associated with iron deficiency. The highest prevalence of both iron deficiency without anemia and anemia was found among children in the South, where only $35.1 \%$ of children had normal iron status; this was followed by Mexico City and its periurban area, with $54.7 \%$. In the North, $61.2 \%$ of children had normal iron status; in the Center, $67.7 \%$ did $(p<0.001)$ (Table 4$)$.

In IO, $62.0 \%$ of children had normal iron status. The rest $(38.0 \%)$ had iron deficiency and/or anemia. Of all cases of anemia found in IO (17.5\%), fewer than half $(7.0 \%)$ were due to iron deficiency; the rest $(10.5 \%)$ were from other causes. In the Center, only $42.9 \%$ of children had normal iron status, compared to $55.0 \%$ of children in the North and $64.9 \%$ of children in the South $(p=0.012)$ (Table 4). There were no statistically significant differences in the overall prevalence of iron deficiency without anemia and anemia between regimens $(p=0.499)$.

\section{Iron status according to hemoglobin and ferritin concentration, by age}

Figure 1 shows data on iron status by age for the whole RR regimen. The largest percentage of normal iron status was found among children younger than 6 months of age. However, in the South, only $64.9 \%$ of children had normal iron status at this age; in Mexico City and its periurban area, $76.6 \%$ did; and in the North and Center, more than $90 \%$ did. We found $9.8 \%$ prevalence of anemia in this age group. It is worth noting that between 6 and 11 months of age, $18.7 \%$ of children had iron deficiency without anemia and $21 \%$ had anemia. Throughout the first year of life, there was a greater prevalence of anemia not associated with iron deficiency as compared with iron-deficiency anemia (7.9 vs. $1.9 \%$ and $13.5 \%$ vs. $7.5 \%$ in children less than 6 months of age and between 6 and 11 months of age, respectively).

Among children in their second year of life, only $46.6 \%$ had normal iron status, with the lowest percentage in the South $(28.4 \%)$. Iron deficiency without anemia in this age group had a prevalence of $29.2 \%$, with an increasing prevalence of iron-deficiency anemia (14.9\%), as compared with $9.3 \%$ of anemia due to other causes.

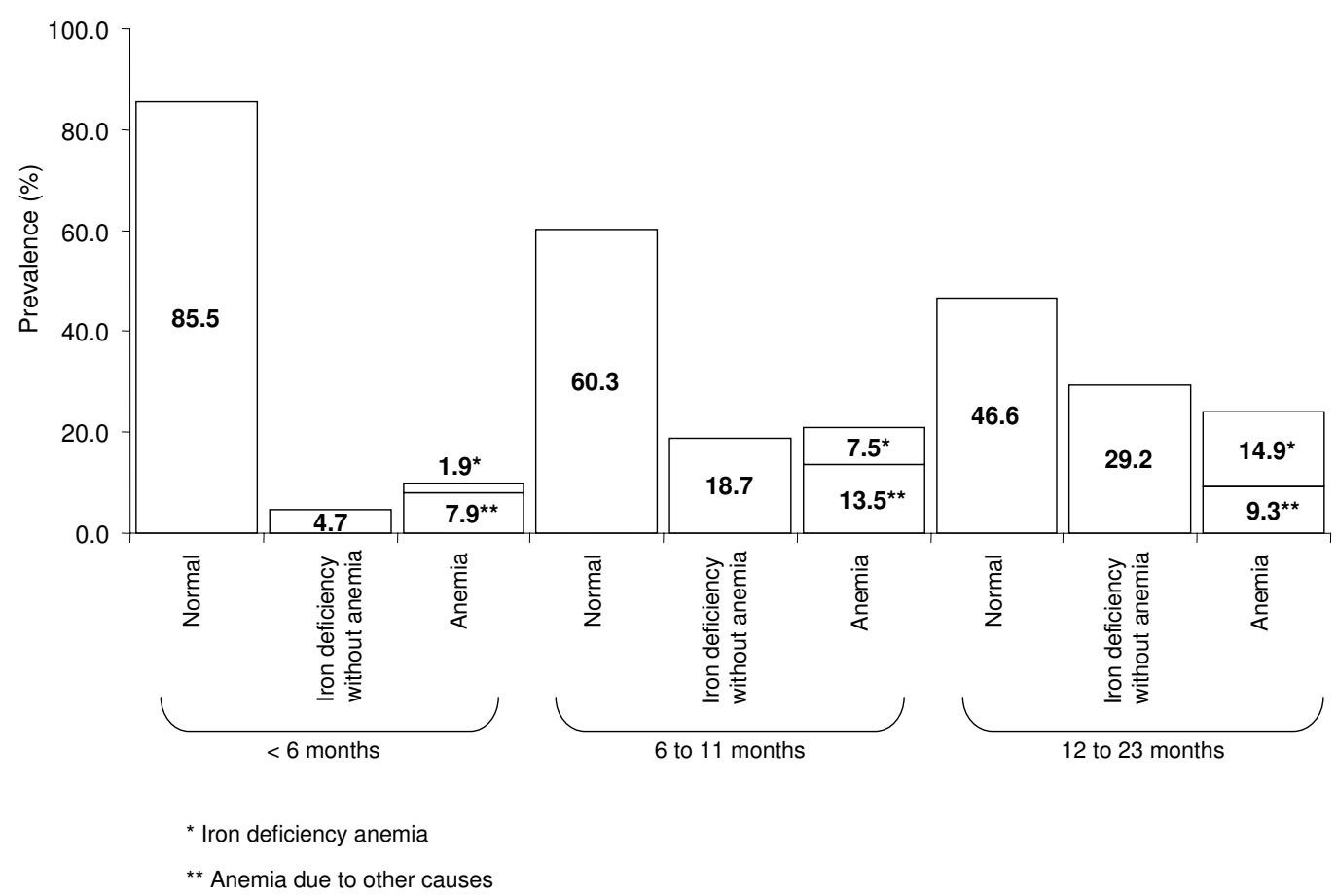

\section{Figure I}

Iron nutritional status according to hemoglobin and ferritin values, by age group, in the regular regimen (RR). Normal: Hemoglobin above the cut-off for anemia and ferritin $>=10 \mu \mathrm{g} / \mathrm{L}$. Iron deficiency without anemia: ferritin $<10 \mu \mathrm{g} / \mathrm{L}$ and $\mathrm{Hb}>=$ cut-off for anemia, by age group. Anemia: $\mathrm{Hb}<$ cut-off for anemia, by age group. Iron deficiency anemia: ferritin < $\mathrm{I} 0 \mu \mathrm{g} / \mathrm{L}$ and $\mathrm{Hb}<$ cut-off for anemia, by age group. Anemia due to other causes: ferritin $\geq 10 \mu \mathrm{g} / \mathrm{L}$ and $\mathrm{Hb}<\mathrm{cut}-\mathrm{off}$ for anemia, by age group. 
Figure 2 shows data on iron status by age for the whole IO regimen. The largest percentage of children with normal iron nutritional status was found among those younger than 6 months of age. However, in the South and Center, only $66.4 \%$ and $69.1 \%$ of children, respectively, had normal iron status at this age, compared to the North, where $84.6 \%$ of children had normal iron status. Prevalence of iron deficiency without anemia even at this early age was $14.6 \%$, with the highest prevalence $(15.9 \%)$ in the South. During the 6 first months of life, prevalence of anemia was $17.4 \%$; however, iron deficiency was associated with anemia only in $5.2 \%$ of cases. In children 6-11 months old, prevalence of iron deficiency without anemia was $23.4 \%$; the prevalence nearly doubled (40.4\%) in the Center. Anemia prevalence was $10.4 \%$, with the highest prevalence $(18.1 \%)$ in the Center. In IO, only $59.6 \%$ of children between 12 and 23 months of age had normal iron status. The lowest prevalence of children with normal iron status $(41.3 \%)$ was found in the Center. Prevalence of iron-deficiency anemia corresponded to $8.5 \% ; 12.1 \%$ corresponded to anemia due to other causes (i.e., associated with normal ferritin values).

\section{Prevalence of folic acid deficiency}

There was a $10.4 \%$ prevalence of folic acid deficiency in RR at a national level; it was highest in the North (15.2\%) and lowest in Mexico City (5.1\%) (Table 5). By age group, children under 6 months old had the highest prevalence (15.1\% at national level). In contrast, children living in IO had an $8.1 \%$ prevalence of folic acid deficiency, with highest in the South $(9.7 \%)$ and lowest in the Center $(1.7 \%)$ (Table 5). Also in contrast to the findings in RR, prevalence of folic acid deficiency was lowest in children under 6 months of age $(0.4 \%)$; folic acid deficiency in this age group was only found in the North. By age group, the highest prevalence was found in children between 12 and 23 months (10.2\%). When comparing between regimens, the difference in the prevalence of folic acid deficiency was not statistically significant $(p=0.72)$.

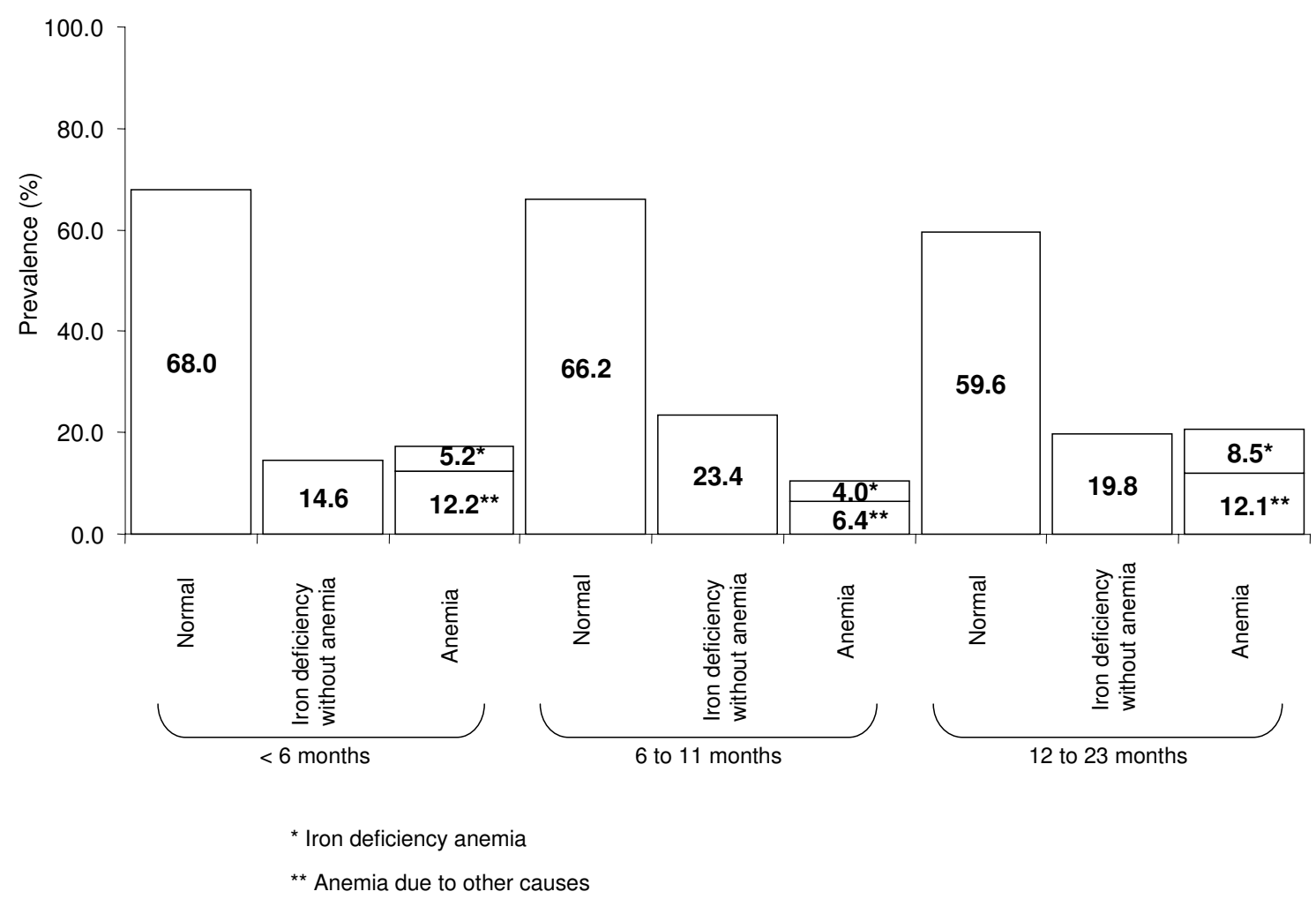

\section{Figure 2}

Iron nutritional status according to hemoglobin and ferritin values, by age group, in IMSS-Oportunidades (IO). Normal: Hemoglobin above the cut-off for anemia and ferritin $>=10 \mu \mathrm{g} / \mathrm{L}$. Iron deficiency without anemia: ferritin < $10 \mu \mathrm{g} / \mathrm{L}$ and $\mathrm{Hb}>=$ cut-off for anemia, by age group. Anemia: $\mathrm{Hb}<$ cut-off for anemia, by age group. Iron deficiency anemia: ferritin < $10 \mu \mathrm{g} / \mathrm{L}$ and $\mathrm{Hb}<$ cut-off for anemia, by age group. Anemia due to other causes: ferritin $\geq 10 \mu \mathrm{g} / \mathrm{L}$ and $\mathrm{Hb}<\mathrm{cut}-\mathrm{off}$ for anemia, by age group. 


\section{Prevalence of low zinc concentrations}

Prevalence of low serum zinc concentrations was $27.9 \%$ in RR and $13.4 \%$ in IO ( $p=0.005)$. The Center had the highest prevalence of low zinc concentration, both in RR $(32.4 \%)$ and in IO (29.9\%). Although there were no statistically significant differences in the prevalence of low zinc concentrations by region in RR, these were statistically significantly different in IO, the highest prevalence found in the Center (29.9\%) and the lowest in the South $(10.4 \%)$. (Table 6).

\section{Multiple micronutrient deficiencies: folic acid and zinc according to iron nutritional status}

Prevalence of folic acid deficiency according to iron nutritional status was not statistically significant different when comparing RR with IO. In RR, children with normal iron status had a prevalence of folic acid deficiency of 8.3\%; children with iron deficiency without anemia had a prevalence of folic acid deficiency of $10.8 \%$; and those with anemia had 19.3\%: folic acid deficiency were found in $18.5 \%$ of children with anemia because of iron deficiency, and $20.1 \%$ was found in children with anemia due to other causes $(p=0.10)$. Similarly, in IO the prevalence of folic acid deficiency was $8.8 \%$ in children with normal iron status, $8.3 \%$ in iron-deficient children without anemia, and $12.8 \%$ in children with anemia: $23.8 \%$ in children with anemia due to iron deficiency and $4.8 \%$ with anemia due to other causes $(p=0.21)$, (Table 7$)$.

Prevalence of low zinc concentrations according to iron status was also not statistically significantly different when comparing RR and IO. Children in RR who showed normal iron status had a $26.1 \%$ prevalence of low zinc concentrations; children with iron deficiency without anemia had a prevalence of $25.8 \%$; those with anemia had $30.2 \%$ prevalence, with $32.7 \%$ in iron-deficiency anemia and $27.8 \%$ in anemia due to other causes $(p=0.44)$. Children in IO with normal iron status had a prevalence of low zinc concentrations in $11.4 \%$ of cases; children with iron deficiency without anemia had low zinc concentration in 9.9\%; children with anemia had 15.1\%: $8.2 \%$ corresponded to children with iron-deficiency anemia and $20.1 \%$ in those with anemia due to other causes $(p=$ 0.24) (Table 7).

In RR, $1.2 \%$ of children had iron, zinc, and folic acid deficiencies simultaneously; $3.0 \%$ had zinc and folic acid deficiencies; $4.3 \%$ had iron and folic acid deficiencies; and $9.2 \%$ had zinc and iron deficiencies. In IO, $0.2 \%$ of children had simultaneous iron, zinc, and folic acid deficiencies; $0.8 \%$ had zinc and folic acid deficiencies; $3.4 \%$ had iron and folic acid deficiencies; and $2.7 \%$ had zinc and iron deficiencies.
In $\mathrm{RR}, 8.2 \%$ of children with anemia had simultaneous zinc and folic acid deficiencies; $7.0 \%$ corresponded to children with iron-deficiency anemia; and 9.4\% corresponded to children with anemia due to other causes.

In $\mathrm{IO}, 0.3 \%$ of children with anemia had simultaneous folic acid and zinc deficiencies: $0.6 \%$ of children had irondeficiency anemia, and $0.1 \%$ had anemia from other causes. Folic acid and zinc deficiencies were present in $0.9 \%$ of children with iron deficiency without anemia and in $1.0 \%$ of children with normal iron status.

\section{Discussion}

The evidence presented in this article is disturbing in terms of public nutrition. It shows that children younger than 2 years old have several micronutrient deficiencies that may stop them from attaining their full growth and development potentials. One of every five children had anemia, and one of every three had iron deficiency. More than half the anemia cases were not accompanied by low ferritin levels, reflecting that they may be from causes other than iron deficiency. Low serum zinc concentrations were found in one out of three children living in the urban areas and in one out of ten of those living in rural areas; $10 \%$ of children of RR and $8 \%$ of children in IO had folic acid deficiencies. Low serum zinc and iron deficiency appeared together in $9 \%$ and $3 \%$ of children living in urban and rural areas, respectively.

Although we have the largest sample size published in the country in this age group, there are several possible sources of bias in our sample. The sample was only representative of children who use the health care services provided by the institution and who were free of acute infectious diseases during the two weeks before giving the blood sample. Although the decision to exclude data about children with acute infectious diseases ensured that the values of iron and zinc would not be affected by the infectious state, it is possible that children who were ill may have had a different micronutrient status than those included in the sample, introducing a further potential bias.

In RR, our study sample was only $64.2 \%$ of the calculated sample size; in IO, it was $74.7 \%$; with differences by regions. In view of the different response rates, which led to underrepresentation of the South and Mexico City regions for $\mathrm{RR}$ and the South region for $\mathrm{IO}$, there is a likelihood of biases in some of the results presented. The different regions in the country have noticeable contrasts in socioeconomic indicators, as the North is more developed than the South. Likewise, the two regimens care for populations with noticeable differences in economic development, favoring the urban areas over the rural/semi-rural. Other possible sources of bias emerge from the composi- 
tion of the subsample of children who participated in the micronutrient study. In comparison with the rest of the children who participated in the larger study, those who were sampled for micronutrient status were older. Also, children in the IO subsample had lower weight for age $\mathrm{Z}$ scores. Therefore, it is possible that the true prevalence might be overestimated, as older children and those with lower weight for age may also be more likely to have micronutrient deficiencies. We also can not rule out the possibility that some characteristics of the study sample might have been influenced by parental acceptance of the study, which might have been affected by their own perceptions and concerns about their children's health status. It is difficult to estimate how the different sampling biases might have influenced the results, however, we are confident that, whatever the direction of the biases, the sampled population reflects the population who use the primary health care facilities of IMSS. To correctly interpret our results, it is necessary to consider some methodological issues. The criterion that we used to define iron deficiency was based only on ferritin values, defining a cutoff of $<10 \mu \mathrm{g} / \mathrm{L}$ to reflect low iron stores. Other indicators of iron status, like the percentage of transferrin saturation (with a cut-off at $<10 \%$ ) were not useful, as it only classified $0.2 \%$ and $3.3 \%$ of children in RR and IO, respectively, as iron deficient. This may be because transferrin saturation has ample variation in the first year of life, so there is no agreement on the best cut-off $[16,30,31]$. In view of the low sensitivity that this indicator has to detect iron deficiency in this age group, we decided not to use it.

There is no general agreement about the preferred cut-off point for ferritin in this age group. We used a cut-off of < $10 \mu \mathrm{g} / \mathrm{L}$, but other authors suggest a different one. Some authors have pointed out that the cut-off values for children under 5 should vary between 8 and $12 \mu \mathrm{g} / \mathrm{L}$, depending on the specific age group [32-36]. WHO [36] recommends using a cut-off of $<12 \mu \mathrm{g} / \mathrm{L}$. We ran our analysis with this WHO-recommended cut-off and found a difference in prevalence of about 4 percentage points higher with it. In NHANES III (1988-1994), the value of the $5^{\text {th }}$ percentile for serum ferritin in children under 1 year of age was equivalent to $11 \mu \mathrm{g} / \mathrm{L}$; the corresponding value for children between 1 and 2 years of age was $6 \mu \mathrm{g} /$ L. However, for the Mexican American sample included in this study, the corresponding values were 8 and $3 \mu \mathrm{g} / \mathrm{L}$, respectively [37]. Looking at healthy Honduran and Swedish children-exclusively breast-fed infants at 4 and 6 months of age-Domellöf et al. found -2 SD cut-off values corresponding at $4 \mathrm{mo}$ of age to $20 \mu \mathrm{g} / \mathrm{L}$, at 6 mo of age to $9 \mu \mathrm{g} / \mathrm{L}$, and at 9 mo of age, to $5 \mu \mathrm{g} / \mathrm{L}$ [38]. Soh et al. have summarized this controversy, stating that until the validity of cut-off points of iron indices is confirmed, prevalence estimates of iron deficiency in children younger than 2 must remain conjectural [39].
Anemia, on the other hand, was defined based on hemoglobin values that were adjusted for each age category in the range of our study. The issue here is whether these cutoffs are adequate to identify anemia. The use of a single cut-off for children under 5 years of age has been a point for discussion [24]. Some authors consider that $11 \mathrm{~g} / \mathrm{dL}$, as proposed by WHO for this age group, may be too high for children less than 2 years old $[16,30,34]$. In fact, changes in iron metabolism found in this age, characterized by a rapid growth spurt and exposure to different infectious diseases, many of which happen subclinically, may affect the biochemical response of the iron status indicators, so there is uncertainty about the proper cutoffs that may best reflect functional outcomes [16,30]. Sheriff et al. found that the 5 th percentile for hemoglobin values for toddlers 8 months of age was $9.7 \mathrm{~g} / \mathrm{dL}$, whereas the corresponding value for children between 12 and 18 months was $10 \mathrm{~g} / \mathrm{dL}$ [34]. Further, a study carried out in Sweden with healthy babies born at term found that more than $30 \%$ had hemoglobin concentrations under $11 \mathrm{~g} / \mathrm{dL}$ by 6 months of age, although fewer than 3\% had ferritin values less than $12 \mu \mathrm{g} / \mathrm{L}$, so few had low iron stores [16].

In our own study, more than $50 \%$ of cases with hemoglobin under the age-specific cut-off were not associated with low ferritin values. It may be that, even when we used cut-off values adjusted for age, we overestimated the prevalence of anemia. The possibility exists that, although we left out any child with data of acute infection and our sample population was mostly drawn from the well-baby clinic and preventive programs, a certain proportion of cases might have had a subclinical infection that raised ferritin values; however, we do not think that this is the most likely explanation. Therefore, children who presented low hemoglobin and normal ferritin values were considered as having anemia from causes other than iron deficiency.

Other micronutrient deficiencies are also known to cause anemia; this is the case of folic acid deficiency, as well as others, like vitamin $\mathrm{A}$ and $\mathrm{B}_{12}$, which were not assessed in our study [40]. Anemia without iron deficiency has been reported by other authors, in some instances in as many as over $50 \%$ of cases, a prevalence similar to the one found in our study $[25,41,42]$. It should be noted that this last figure also holds true when we analyzed our data using a cut-off of $<12 \mu \mathrm{g} / \mathrm{L}$ for serum ferritin to identify iron deficiency.

Even when most of the Mexican population is covered by the IMSS system, this population has several different characteristics. On the one hand, for $\mathrm{RR}$, at least one of the parents has to have a regular salary to be an IMSS beneficiary. On the other hand, the populations served by IO are also beneficiaries of other subsidy and nutrition allevia- 
tion government programs, such as Oportunidades [43]. This and other programs are targeted at low-income households, and include the provision of micronutrientfortified products for pregnant/lactating women and infants between the ages of 4 and 23 months old, as well as for underweight children aged 2-4 and may have some influence in improving the micronutrient status of these groups [44]. Thus, it is relevant to compare our data with those collected by the NNS conducted in 1999, in Mexico as both studies were carried out at approximately the same time. However, there were some methodological differences: our survey used venous blood for sampling, whereas NNS used capillary samples; our study used ageadjusted hemoglobin cut-offs values to identify anemia, whereas NNS used two cut-offs: $9.5 \mathrm{~g} / \mathrm{dL}$ for children 611 months old (compared to $10.5 \mathrm{~g} / \mathrm{dL}$ used in the present study for this age group), and $11 \mathrm{~g} / \mathrm{dL}$ for children 12-23 months old (compared to $10.7 \mathrm{~g} / \mathrm{dL}$ for this age group in our study) [13].

With these caveats in mind, we noted that children 6-11 months old in RR had a prevalence of anemia of 20.9 $(95 \% \mathrm{CI}=17.0-25.4)$, higher than that found by NNS: $11.3 \%(95 \% \mathrm{CI}=11.2-21.0)$. In comparison, the prevalence found in children in IO was very similar to the one found in the NNS rural areas of the country: $15.5 \%, 95 \%$ $\mathrm{CI}=11.2-21.0$ vs. $16.2 \%, 95 \% \mathrm{CI}=11.8-20.6$, respectively. In our study, children between 12 and 23 months old had lower prevalence of anemia than the general Mexican population reported by NNS, both in the RR and the IO areas: $22.7 \%, 95 \% \mathrm{CI}=19.2-26.6$ vs. $46.8 \%, 95 \% \mathrm{CI}$ $=43.1-50.5$ in urban areas and $21.7 \%, 95 \% \mathrm{CI}=17.3-$ 27.0 vs. $52.9 \%, 95 \% \mathrm{CI}=48.0-57.9$, for the IMSS and the general population, respectively. Taking an external reference as comparison, in the 2002 report of the WHO for Latin America, the prevalence of anemia in children under 5 varied between $16 \%$ and $28 \%$ [45]. Although the prevalence that we found in our study lies well within this range, it is known that the prevalence of anemia is higher in the first 2 years of life. Therefore, the prevalence that we found in our population may be lower than that in other Latin American countries and was lower than that found in the general Mexican population.

Another interesting comparison was found when contrasting iron deficiency between RR and IO. In both regimens, iron deficiency without anemia and anemia were present since the first 6 months of life in 15\% of children under RR and $32 \%$ of children under IO. Iron deficiency at this early age has often been found to be related to a combination of the poor iron stores accumulated over the last trimester of pregnancy and the poor quality of complementary foods, which may be either low in iron or in sources of highly bio-available iron $[18,39]$. Further, it was clear that the prevalence of iron deficiency increased with age, a fact that calls attention to the need for early actions by primary health care public services.

With respect to other micronutrient deficiencies, the only statistical significant difference between RR and IO was a higher prevalence of low serum zinc concentration in the former. Although it would be expected to find higher prevalences of micronutrient deficiencies in the more underprivileged areas, the targeted food and nutritional assistance that the underserved population has received over the past years may be reflected in an improvement in their micronutrient status [44]. It is generally accepted that we do not have good indicators to assess zinc nutritional status, especially as related to biological responses that may translate functional outcomes of mild to moderate deficiency. Some studies have evaluated the adequacy of dietary consumption, whereas others have used plasma or serum concentration of zinc. The WHO has estimated the prevalence of zinc deficiency based on dietary intake, which has a mean of $31 \%$ worldwide, ranging between 6 and $73 \%$ in different populations in the under-5-year-old group [45]. The most widely used indicator, however, has been plasma or serum zinc concentration $[7,26]$. Using this indicator, we found a high prevalence of zinc deficiency, particularly in children of RR. Even when the consequences of zinc deficiency have been fairly well documented in the literature and include limitations on growth potential, impaired psycho-motor development, impaired immune function, and delayed bone and sexual maturation onset $[5-7,46,47]$, the extent of zinc deficiency has not been properly established worldwide, nor are there specific public nutrition programs addressing it, aside from the recent zinc supplementation recommended for children with acute diarrhea $[5,46,48]$.

Another result worth discussing is the presence of multiple micronutrient deficiencies. When compared to children with normal iron status, children with anemia had a higher prevalence of folic acid deficiency. Similarly, low plasma zinc concentrations were more prevalent in children with anemia. Although the prevalence of children with three simultaneous deficiencies of the micronutrients studied was relatively low, the coincidence of two of these deficiencies singled out those of zinc and iron as the most common deficiencies in RR children, and iron and folic acid in IO. These deficiencies will manifest themselves as anemia-not necessarily related to iron deficiency. They call for more comprehensive interventionswhether food based, supplement based, or fortification based-than the ones usually considered by public health programs. Further, the early age at which these deficiencies present themselves is a call for public health interventions to consider preventive approaches rather than curative ones. 


\section{Conclusion}

Iron and zinc are the principal micronutrient deficiencies in Mexican children younger than 2 years old who use the health care services provided by IMSS. Anemia not associated with low ferritin values was more prevalent than iron-deficiency anemia. The fact that we found multiple micronutrient deficiencies at this early age reveals a need to establish effective preventive public health nutrition programs to address them.

\section{Competing interests}

The author(s) declare that they have no competing interests.

\section{Authors' contributions}

The authors' contributions to this publication are as follows: designing the study, analyzing the data, writing the manuscript, and obtaining funding for this research (MXDL, SFH, SFH, IMR, SM, HMS); supervising the project, evaluating data, reviewing the draft, and contributing comments for the final manuscript (MEM, BT, GMA, RIR, MGU). All authors read and approved the final manuscript.

\section{Acknowledgements}

The authors recognize the participation of Dr. José Olvera and Dr. Marcela Valverde, from IMSS-Oportunidades, who collaborated with the sample design and coordinated the field study in IO. They also appreciate the collaboration of Dr. Salvador Villalpando, who gave advice as to the chemical determinations and laboratory techniques used in the analyses, and of chemists Guadalupe Matute, Irene Montalvo, Rosa Imelda Moreno, Catalina Ramírez, and Roberto Cervantes for their careful laboratory determinations. Nurse Rosa Alicia García helped organize lab samples and the database. Analysts Vanessa Hernández and Javier Montaño spent a great deal of time designing, receiving, and putting together the databases. The research team also wants to acknowledge fieldworkers and lab technicians at the primary health care units who participated in the study. Special thanks are due to the children and parents whose participation made this study possible.

The project received funding from CONACYT, project. No. 3877I-M as well as from Fondo para el Fomento de la Investigación Médica-IMSS, project numbers FP-0245 and FP-200I/257.

\section{References}

I. Allen LH: The nutrition CRSP: what is marginal malnutrition, and does it affect human function? Nutr Rev 1993, 5 I (9):255-267.

2. Pollitt E, Saco-Pollitt C, Leibel RL, Viteri FE: Iron deficiency and behavioral development in infants and preschool children. Am J Clin Nutr 1986, 43(4):555-565.

3. Walter T, De Andraca I, Chadud P, Perales CG: Iron deficiency anemia: adverse effects on infant psychomotor development. Pediatrics 1989, 84(1):7-17.

4. Allen LH: Nutritional influences on linear growth: a general review. Eur J Clin Nutr 1994, 48(SuppI I):S75-S89.

5. Bhutta ZA, Black RE, Brown KH, Gardner JM, Gore S, Hidayat A, Khatun F, Martorell R, Ninh NX, Penny ME, Rosado JL, Roy SK, Ruel $M$, Sazawal S, Shankar A: Prevention of diarrhea and pneumonia by zinc supplementation in children in developing countries: pooled analysis of randomized controlled trials. Zinc Investigators' Collaborative Group. J Pediatr 1999, 135(6):689-697.
6. Salgueiro MJ, Zubillaga M, Lysionek A, Cremaschi G, Goldman CG, Caro R, De Paoli T, Hager A, Weill R, J. B: Zinc status and immune system relationship: a review. Biol Trace Elem Res 2000, 76(3): 193-205.

7. Brown $\mathrm{KH}$, Wuehler SE, Peerson JM: The importance of zinc in human nutrition and estimation of the global prevalence of zinc deficiency. Food and Nutrition Bulletin 200I, 22(2): I I3-I 25.

8. Dutta P, Mitra U, Datta A, Niyogi SK, Dutta S, Manna B, Basak M, Mahapatra TS, Bhattacharya SK: Impact of zinc supplementation in malnourished children with acute watery diarrhoea. J Trop Pediatr 2000, 46(5):259-263.

9. Umeta M, West CE, Haidar J, Deurenberg P, Hautvast JG: Zinc supplementation and stunted infants in Ethiopia: a randomised controlled trial. Lancet 2000, 355(9220):2021-2026.

10. Fishman SM, Christian P, West KP: The role of vitamins in the prevention and control of anaemia. Public Health Nutr 2000, 3(2): $125-150$.

II. Osorio MM, Lira PI, Batista-Filho M, Ashworth A: Prevalence of anemia in children 6-59 months old in the state of Pernambuco, Brazil. Rev Panam Salud Publica 200I, I 0(2): I0I-107.

12. Sharmanov A: Anaemia in Central Asia: Demographic and health survey experience. Food and Nutrition Bulletin 1998, 19(4):307-317.

13. Villalpando S, García-Guerra A, Ramírez-Silva Cl, Mejía-Rodríguez F, Matute G, Shamah-Levy T, Rivera JA: Iron, zinc and iodide status in Mexican children under 12 years and women $12-49$ years of age. A probabilistic national survey. Salud Publica Mex 2003, 45 (Suppl 4):S520-S529.

14. Villalpando S, Montalvo-Velarde I, Zambrano N, García-Guerra A, Ramírez-Silva Cl, Shamah-Levy T, Rivera JA: Vitamins A, and $C$ and folate status in Mexican children under 12 years and women 1 2-49 years: a probabilistic national survey. Salud Publica Mex 2003, 45(suppl 4):S508-5I9.

15. Villalpando S, Shamah-Levy T, Ramírez-Silva Cl, Mejía-Rodríguez F, JA. R: Prevalence of anemia in children $I$ to 12 years of age. Results from a nationwide probabilistic survey in Mexico. Salud Publica Mex 2003, 45(Suppl 4):S490-S498.

16. Aggett PJ, Agostoni C, Axelsson I, Bresson JL, Goulet O, Hernell O, Koletzko B, Lafeber HL, Michaelsen KF, Micheli JL, Rigo J, Szajewska $\mathrm{H}$, Weaver LT: Iron metabolism and requirements in early childhood: do we know enough? A commentary by the ESPGHAN Committe on Nutrition. J Pediatr Gastroenterol Nutr 2002, 34(4):337-345.

17. Zetterström R: Iron deficiency and iron deficiency anaemia during infancy and childhood. Acta Paediatr 2004, 93(4):436-439.

18. Flores-Huerta S, Martínez H: Prácticas de alimentación, estado de nutrición y cuidados a la salud en niños menores de 2 años en México. Atendidos por el Instituto Mexicano del Seguro Social. Mexico City; 2004.

19. Sepúlveda A, Lezana M, Tapia C, Valdespino J, Madrigal H, Kumate J: Estado nutricional de preescolares y mujeres en México: resultados de una encuesta probabilística nacional. Gac Med Mex 1990, I 26(3):207-224.

20. Rivera-Dommarco J, Shamah-Levy T, Villalpando-Hernández S, González-de Cossío T, Hernández-Prado B, Sepúlveda J: Encuesta Nacional de Nutrición 1999. Estado nutricio de niños y mujeres en México . In Encuestas de Salud Cuernavaca, Morelos, Instituto Nacional de Salud Pública; 200I.

21. O'Broin SD, Gunther EW: Screening of folate status with use of dried blood spots on filter paper. Am J Clin Nutr 1999, 70(3):359-367.

22. Ruiz-Argüelles GJ, Llorente-Peters A: Predictive equations for normal red cell values at altitudes from sea level to 2,670 meters. Rev Invest Clin 1981, 33(2): 19|-193.

23. Brugnara C: References values in Infancy and Childhood. In Hematology of Infancy and Childhood Volume I. 5th edition. Edited by: Oski FA, Brugnara C, Nathan D. Philadelphia , W.B. Saunders Company; 1993:i-xxviii.

24. World Health Organization: Report of WHO/UNICEF/UNU Consultation on indicators and strategies for iron deficiency and anemia programmes. In WHO/UNICEFIUNU CONSULTATION Geneva, WHO; 1994.

25. Male C, Persson LA, Freeman V, Guerra A, van't Hof MA, Haschke F, Euro-Growth Iron Study Group: Prevalence of iron deficiency in I2-mo-old infants from II European areas and influence of 
dietary factors on iron status (Euro-Growth study). Acta Paediatr 200I, 90(5):492-498.

26. Hotz C, Peerson JM, Brown KH: Suggested lower cutoffs of serum zinc concentrations for assessing zinc status: reanalysis of the second National Health and Nutrition Examination Survey data (1976-1980). Am / Clin Nutr 2003, 78(4):756-764.

27. Shackman G: Sample size and design effect. 200I [http://fac ulty.smu.edu/slstokes/stat6380/deff\%20doc.pdf]. Presented at Albany Chapter of American Statistical Association, March 200I. Accessed 16 August 2007

28. Sturgis P: Analysing Complex Survey Data: Clustering, Stratification and Weights. Social Research UPDATE 2004:2-5.

29. Stata Corporation 4905 Lakeway Drive: STATA-PC. In College Station Special Edition. 8.2 Texas 77845 USA; 2005.

30. Hernell $O$, Lönnerdal $B$ : Is iron deficiency in infants and young children common in Scandinavia and is there a need for enforced primary prevention? Acta Paediatr 2004, 93(8): 1024-1026.

31. Saarinen UM, Siimes MA: Developmental changes in serum iron, total iron-binding capacity, and transferrin saturation in infancy. J Pediatr 1977, 9 I(6):875-877.

32. Demaeyer EM, Dallman P, Gurney JM, Hallberg GL, Sood SK, Srikantia SG: Preventing and Controlling Iron Deficiency Anemia through Primary Health Care: A Guide for Health Administrators and Programme Managers. World Health Organization; 1989:8-9.

33. Emond AM, Hawkins N, Pennock C, Golding J: Haemoglobin and ferritin concentrations in infants at 8 months of age. Arch Dis Child 1996, 74(I):36-39.

34. Sherriff A, Emond A, Hawkins N, Golding J: Haemoglobin and ferritin concentrations in children aged 12 and 18 months. ALSPAC Children in Focus Study Team. Arch Dis Child 1999, 80(2):153-157.

35. Soh P, Ferguson EL, McKenzie JE, Skeaff S, Parnell W, Gibson RS: Dietary intakes of 6-24-month-old urban South Island New Zealand children in relation to biochemical iron status. Public Health Nutr 2002, 5(2):339-346.

36. UNICEF/UNU/WHO Consultation: Iron deficiency anaemia: Assessment, prevention and control. A guide for programme managers. World Health Organization; 2001:38.

37. Hollowell JG, van Assendelft OW, Gunter EW, Lewis BG, Najjar M, Pfeiffer C. Centers for Disease Control and Prevention, National Center for Health Statistics: Hematological and iron-related analytes. Reference data for persons aged I year and over: United States, 1988-94. In Vital Health Stat II Hyattsville, Maryland, National Center for Health Statistics; 2005:I 36.

38. Domellöf M, Dewey KG, Lönnerdal B, Cohen RJ, Hernell O: The diagnostic criteria for iron deficiency in infants should be reevaluated. J Nutr 2002, I 32( I 2):3680-3686.

39. Soh P, Ferguson EL, McKenzie JE, Homs MY, Gibson RS: Iron deficiency and risk factors for lower iron stores in 6-24-monthold New Zealanders. Eur J Clin Nutr 2004, 58(I):7I-79.

40. Allen LH, Rosado JL, Casterline JE, Martinez H, Lopez P, Muñoz E, Black AK: Vitamin B-I2 deficiency and malabsorption are highly prevalent in rural Mexican communities. Am J Clin Nutr 1995, 62(5):1013-1019.

41. Stoltzfus RJ: Defining iron-deficiency anemia in public health terms: a time for reflection. J Nutr 200I, I 3 I (2S-2):565S-567S.

42. Stoltzfus RJ: Iron-deficiency anemia: reexaminig the nature and magnitude of the public health problem. Summary: implications for research and programs. J Nutr 200I, I3 I(2S2):697S-70IS

43. Secretaría de desarrollo Social: Más oportunidades para las familias pobres: Evaluación de resultados del Programa de Educación, Salud y Alimentación. Primeros avances, 1999. Mexico City ; 1999:397.

44. Rivera JA, Sotres-Alvarez D, Habicht JP, Shamah T, Villalpando S: Impact of the Mexican program for education, health, and nutrition (Progresa) on rates of growth and anemia in infants and young children: a randomized effectiveness study. JAMA 2004, 29 I(2 I):2563-2570.

45. World Health Organization: The world health report 2002. Reducing Risks, Promoting Healthy Life. In The world health report World Health Organization; 2002.
46. Sandström B: Diagnosis of zinc deficiency and excess in individuals and populations. Food and Nutrition Bulletin 200I, 22(2): $133-136$.

47. Scott ME, Koski KG: Zinc deficiency impairs immune responses against parasitic nematode infections at intestinal and systemic sites. J Nutr 2000, I30(5S Suppl): |4|2S-1420S.

48. Penny ME, Marin RM, Duran A, Peerson JM, Lanata CF, Lönnerdal B, Black RE, Brown KH: Randomized controlled trial of the effect of daily supplementation with zinc or multiple micronutrients on the morbidity, growth, and micronutrient status of young Peruvian children. Am J Clin Nutr 2004, 79(3):457-465.

\section{Pre-publication history}

The pre-publication history for this paper can be accessed here:

\section{http://www.biomedcentral.com/1471-2458/7/345/pre} pub
Publish with Biomed Central and every scientist can read your work free of charge

"BioMed Central will be the most significant development for disseminating the results of biomedical research in our lifetime. "

Sir Paul Nurse, Cancer Research UK

Your research papers will be:

- available free of charge to the entire biomedical community

- peer reviewed and published immediately upon acceptance

- cited in PubMed and archived on PubMed Central

- yours - you keep the copyright

Submit your manuscript here:

http://www.biomedcentral.com/info/publishing_adv.asp
BioMedcentral 